\title{
Search for a light pseudoscalar Higgs boson produced in association with bottom quarks in pp collisions at $\sqrt{s}=8 \mathrm{TeV}$
}

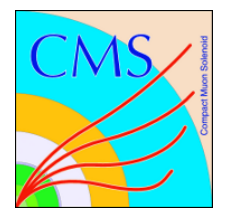

\section{The CMS collaboration}

E-mail: cms-publication-committee-chair@cern.ch

Abstract: A search for a light pseudoscalar Higgs boson (A) produced in association with bottom quarks and decaying into a muon pair is reported. The search uses $19.7 \mathrm{fb}^{-1}$ of proton-proton collisions at a center-of-mass energy of $8 \mathrm{TeV}$, collected by the CMS experiment. No signal is observed in the dimuon mass range from 25 to $60 \mathrm{GeV}$. Upper limits on the cross section times branching fraction, $\sigma(\mathrm{pp} \rightarrow \mathrm{b} \overline{\mathrm{b}} \mathrm{A}) \mathcal{B}(\mathrm{A} \rightarrow \mu \mu)$, are set.

KEYwORDS: Hadron-Hadron scattering (experiments), Higgs physics

ARXIV EPRINT: 1707.07283 


\section{Contents}

1 Introduction 1

2 The CMS detector 2

3 Data and simulated samples $\quad 2$

$4 \quad$ Event reconstruction and selection 3

5 Results and systematic uncertainties 5

$\begin{array}{llr}6 & \text { Summary } & 10\end{array}$

$\begin{array}{lr}\text { The CMS collaboration } & 16\end{array}$

\section{Introduction}

The CMS and ATLAS experiments at the CERN LHC have made clear observations of a particle compatible with the standard model (SM) Higgs boson [1-4]. While the observation serves as a powerful affirmation of the SM mechanism for generating particle masses, the discovery does not rule out the existence of a more complex theory with an extended Higgs sector. The discovery of additional scalar or pseudoscalar bosons may be evidence of such a scenario.

A number of well-motivated models extending the SM predict the existence of new Higgs bosons. One well studied scenario is the Two Higgs Doublet Model (2HDM) $[5,6]$ predicting the existence of additional elementary Higgs particles. The 2HDM includes a CP-odd Higgs boson, A, that could be light and produced in association with bottom quarks at the LHC. In some schemes, with a negatively-signed Yukawa coupling to the down-type fermions, the associated $b \bar{b} A$ production cross section times branching fraction into muons can be very large for an A boson with a mass below about $60 \mathrm{GeV}$, i.e. less than half of the mass of the observed SM-like Higgs boson [7]. It can vary from 1000 to $35 \mathrm{fb}$ for $\mathrm{A}$ boson masses in the range from 25 to $60 \mathrm{GeV}$ at a center-of-mass energy of $8 \mathrm{TeV}$ in proton-proton collisions.

We present a search for muon pairs, in the mass range from 25 to $60 \mathrm{GeV}$, produced in association with bottom quarks in the context of the $2 \mathrm{HDM}$. The search uses $19.7 \mathrm{fb}^{-1}$ of data collected by the CMS experiment in proton-proton collisions at a center-of-mass energy of $8 \mathrm{TeV}$. The analysis of the $\mu \mu$ final state complements the CMS searches for a low-mass A boson decaying into $\tau$ lepton pairs by the CMS Collaboration [8,9]. Despite the significantly lower branching fraction, the $\mu \mu$ final state profits from much better dilepton mass resolution as well as higher trigger and lepton identification efficiencies. This is the 
first time associated production with $\mathrm{b}$ quarks in the dimuon decay channel has been searched for in the low dimuon mass range.

In the following, sections 2 and 3 describe the CMS detector, the data, and simulated samples. The event reconstruction and selections are presented in section 4 . In section 5 , the result of the search for the $\mathrm{b} \overline{\mathrm{b}} \mathrm{A}$ process is presented. The paper is summarized in section 6 .

\section{The CMS detector}

The central feature of the CMS apparatus [10] is a superconducting solenoid, $13 \mathrm{~m}$ in length and $6 \mathrm{~m}$ in diameter, which provides an axial magnetic field of $3.8 \mathrm{~T}$. Within the field volume there are several particle detection systems. Charged-particle trajectories are measured by silicon pixel and strip trackers, covering $0 \leq \phi \leq 2 \pi$ in azimuth and $|\eta|<2.5$ in pseudorapidity. A lead tungstate crystal electromagnetic calorimeter (ECAL) surrounds the tracking volume. It is comprised of a barrel region $|\eta|<1.48$ and two endcaps that extend up to $|\eta|=3$. A lead and silicon-strip preshower detector is located in front of the ECAL endcaps. A brass and scintillator hadron calorimeter surrounds the ECAL and also covers the region $|\eta|<3$. Steel forward calorimeters with quartz fibers, read out by photomultipliers, extend the calorimetric coverage up to $|\eta|=5$. Muons are identified and measured in gas-ionization detectors embedded in the steel flux-return yoke outside the solenoid. The detector is nearly hermetic, allowing energy balance measurements in the plane transverse to the beam direction.

\section{Data and simulated samples}

A two-tier trigger system selects collision events of interest. For this analysis, events are first selected by requiring a single muon $(\mu)$ or two muons within the detector acceptance and passing loose identification and kinematic requirements. For the single-muon trigger, the muon must have $|\eta|<2.1$. The muon is required to be isolated and must have transverse momentum $\left(p_{\mathrm{T}}\right)$ greater than $24 \mathrm{GeV}$. The dimuon trigger requires two muons with $p_{\mathrm{T}}$ greater than $17 \mathrm{GeV}$ for the leading muon and $8 \mathrm{GeV}$ for the sub-leading muon. The dielectron trigger requires two electrons with $p_{\mathrm{T}}$ greater than $17 \mathrm{GeV}$ for the leading electron and $8 \mathrm{GeV}$ for the sub-leading electron and the electrons are required to be isolated.

The analysis uses opposite-sign dimuon events with additional jets, with at least one of them being identified as originating from b quark fragmentation ("b jet"). The invariant mass of the opposite-sign lepton pair $\left(m_{\mu \mu}\right)$ is required to be greater than $12 \mathrm{GeV}$ to reject low-mass resonances and remove poorly-modeled backgrounds.

The Monte Carlo (MC) simulation is used to optimize the event selection to give the best sensitivity for signal discovery. Predictions for the yields of background processes, which are also based on simulation, are validated using data in appropriate control regions. The pp $\rightarrow$ b $\bar{b} A$ signal events are simulated with PYTHIA (v6.4.26) [11]. The signal samples have been generated for A masses in intervals of $10 \mathrm{GeV}$. The natural width of the A boson has been set to a value of less than $50 \mathrm{MeV}$, which is much smaller than the dimuon mass resolution in the mass range from 25 to $60 \mathrm{GeV}\left(\sigma_{\mu \mu}=0.45 \mathrm{GeV}\right.$ for $\left.m_{\mathrm{A}}=30 \mathrm{GeV}\right)$. 
The following background processes have been considered: Drell-Yan (DY), W+jets, $\mathrm{t} \overline{\mathrm{t}}$, single top quark, and diboson production.

The DY events are simulated at leading order (LO) using the MADGRAPH (v5.1.3.30) generator $[12,13]$ interfaced to PYTHIA (v6.4.26) for parton showering and hadronization. The CTEQ6L1 [14] parton distribution functions (PDFs) are used to generate the events. The MADGRAPH generation includes up to four partons in the matrix element calculations and a procedure to properly merge jets from the matrix element calculations and parton shower $[15,16]$. The DY sample is normalized to the next-to-next-to-leading order (NNLO) cross section computed with FEWZ (v3.1) [17]. The W+jets sample is simulated and normalized in a similar way to the DY+jets sample. The $\ell \ell(\ell \nu)$ production in association with $\mathrm{b}$ and $\mathrm{c}$ quarks is included in the DY+jets $(\mathrm{W}+$ jets $)$ samples.

Top quark pair events are generated with MADGRAPH, including up to three extra partons, and the simulated sample is normalized to the NNLO+NNLL (next-to-next-toleading-logarithmic) inclusive cross section [18]. Single top quark processes (t, s, and Wt channels) are modeled at next-to-leading order (NLO) with POWHEG [19-21] and normalized to the approximate NNLO cross sections [22, 23]. Diboson production processes (VV) $\mathrm{WW}, \mathrm{WZ} / \gamma^{*}$ and $\mathrm{Z} / \gamma^{*} \mathrm{Z} / \gamma^{*}$ are generated with MADGRAPH and normalized, respectively, to their NLO cross sections $[24,25]$. The $\mathrm{WZ} / \gamma^{*}$ and $\mathrm{Z} / \gamma^{*} \mathrm{Z} / \gamma^{*}$ processes are generated with $m_{\gamma^{*}}>10 \mathrm{GeV}$.

The PYTHIA parameters for the underlying event are set according to the Z2* tune [26, 27], an update of the $\mathrm{Z} 1$ tune described in refs. [28, 29].

A detector simulation based on GEANT4 (v.9.4p03) [30, 31] is applied to all the generated signal and background samples. The presence of multiple pp interactions (pileup) in the same or adjacent bunch crossings is incorporated by simulating additional interactions (both in-time and out-of-time with the collision) with a multiplicity distribution that matches the one observed in data. The average number of pileup events is estimated as 21 interactions per bunch crossing. The observed jet energy resolution and jet energy scale measurements [32], b tagging efficiency and b tagging discriminator distributions [33] in data are used to correct the simulated events.

\section{Event reconstruction and selection}

Events are required to have at least one vertex, with the reconstructed longitudinal position $(z)$ within $24 \mathrm{~cm}$ of the geometric center of the detector and the transverse position within $2 \mathrm{~cm}$ of the beam interaction region. In the case of multiple reconstructed vertices associated with additional pp interactions, the one with the highest scalar sum of the $p_{\mathrm{T}}^{2}$ of its associated tracks is chosen as the primary vertex. Muons are required to originate from the same primary vertex by requiring the longitudinal and transverse impact parameters with respect to the primary vertex to be less than $0.5 \mathrm{~cm}$ and $0.2 \mathrm{~cm}$, respectively.

Muon candidates are reconstructed with a global trajectory fit using hits in the tracker and the muon system [34]. The efficiency for muons to pass both the identification and isolation requirements is measured to be more than $95 \%$ for the kinematic region studied in this analysis [3]. 
The electron selection criteria are optimized using a multivariate approach, and have a combined identification and isolation efficiency of approximately $60 \%$ at low $p_{\mathrm{T}}(\approx 10 \mathrm{GeV})$ and $90 \%$ at high $p_{\mathrm{T}}(>50 \mathrm{GeV})$ for electrons from $\mathrm{W}$ or $\mathrm{Z}$ boson decays [35]. The training of the multivariate electron reconstruction is performed using simulated events, while the performance is validated using data.

In the interest of distinguishing between prompt and nonprompt lepton candidates (predominantly arising from decays of $\mathrm{b}$ hadrons), a relative isolation is defined for each lepton candidate. The muon isolation variable is computed as the sum of the transverse momenta of the charged particles inside a cone of radius $\Delta R \equiv \sqrt{(\Delta \eta)^{2}+(\Delta \phi)^{2}}=0.3$ around the lepton direction divided by the muon $p_{\mathrm{T}}$. The isolation is required to be smaller than 0.1. If the signal muons are within a distance of $\Delta R<0.3$ of each other the $p_{\mathrm{T}}$ of one lepton is subtracted from the isolation value of the other lepton.

The anti- $k_{\mathrm{T}}$ clustering algorithm $[36,37]$ with a distance parameter of 0.5 is used for jet reconstruction.

Two types of jets are used in the analysis, "jet-plus-track" (JPT) [38] and particleflow (PF) [39] jets. The two algorithms combine the information from all subdetectors. However, the PF algorithm separates deposits in the calorimeter into clusters and matches clusters with reconstructed tracks (PF candidates), while the JPT algorithm does not require cluster separation as it starts from the reconstructed calorimeter jets (calojet) and track matching is done with respect to the calojet area at the surface of the calorimeter. Also the algorithms have a different approach to the calibration of the constituents: cluster calibration from simulation in the case of the PF algorithm; and corrections due to calorimeter thresholds and tracking inefficiency in the case of the JPT algorithm. These jet reconstruction algorithms are affected differently by detector effects, like tracker misalignment or the calibration of the calorimeter, and thus serve as an important cross-check of each other. The two types of jet, JPT and PF, provide similar jet energy resolution. The final results are presented with $\mathrm{PF}$ jets.

Jets with a significant fraction of energy coming from pileup interactions or not associated with the primary vertex are rejected [38, 40]. The remaining pileup energy in jets is subtracted using a technique that relies on information about the jet area [37, 41, 42]. Jets are required to satisfy identification criteria that remove jets originating from noisy channels in the calorimeters $[43,44]$. Calibrated jets $[32,45]$ are required to have $p_{\mathrm{T}}>20 \mathrm{GeV}$, $|\eta|<4.7$ and to be separated by at least 0.5 in $\Delta R$ from muons passing the selection requirements described above.

The missing transverse momentum $\left(p_{\mathrm{T}}^{\text {miss }}\right)$ is defined as the modulus of the vector sum of the transverse momenta of all reconstructed PF candidates in the event. The calibrations applied to jets are propagated to the $p_{\mathrm{T}}^{\mathrm{miss}}$.

Jets originating from the hadronization of $\mathrm{b}$ (or c) quarks are identified by a multivariate analysis [46] based on a secondary vertex b tagging algorithm described in refs. [33, 47]. The algorithm combines information on track impact parameter significance, secondary vertices, and jet kinematics. Several working points are available; these are designed to yield successively higher purity at the cost of reduced efficiency for identifying b jets. The working point used in this analysis corresponds to a b tagging identification efficiency of 
$45 \%$ and a misidentification rate of $0.05 \%$ for light-flavor $(\mathrm{u}, \mathrm{d}, \mathrm{s}, \mathrm{g})$ jets with $p_{\mathrm{T}}>30 \mathrm{GeV}$. The misidentification rate for $\mathrm{c}$ jets is $\simeq 6 \%$.

Selections are optimized using simulated events for the signal model $\mathrm{pp} \rightarrow \mathrm{b} \overline{\mathrm{b}} \mathrm{A}, \mathrm{A} \rightarrow$ $\mu \mu$ with $m_{\mathrm{A}}=30 \mathrm{GeV}$ and simulated backgrounds. The threshold on the leading muon $p_{\mathrm{T}}^{\mu_{1}}$ is fixed to $25 \mathrm{GeV}$. This choice is determined by the single-muon trigger $p_{\mathrm{T}}$ threshold of $24 \mathrm{GeV}$. The rapidity requirements on the muons and $\mathrm{b}$ jets are driven by the trigger and particle identification conditions. The optimization procedure selects the thresholds on the subleading $p_{\mathrm{T}}$ muon $\left(p_{\mathrm{T}}^{\mu_{2}}\right)$, the leading $\mathrm{b}$ jet $p_{\mathrm{T}}\left(p_{\mathrm{T}}^{\mathrm{b} \text { jet }}\right)$, and $p_{\mathrm{T}}^{\text {miss }}$, where an approximate estimate of signal significance, defined as $\mathrm{Z}=2(\sqrt{S+B}-\sqrt{B})$ [48] reaches its maximal

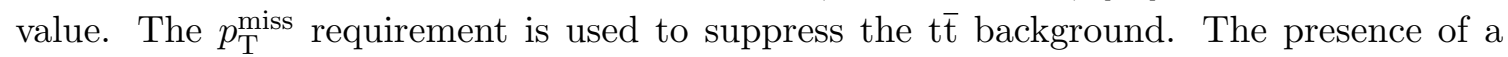
second jet (b tagged or not) is not required since in the $\mathrm{b} \overline{\mathrm{b}} \mathrm{A}$ production process the second $\mathrm{b}$ jet is often at high $\eta$ or has too low $p_{\mathrm{T}}$.

Optimization in this way gives rise to:

- $p_{\mathrm{T}}^{\mu_{1}}>25 \mathrm{GeV},\left|\eta_{\mu_{1}}\right|<2.1$

- $p_{\mathrm{T}}^{\mu_{2}}>5 \mathrm{GeV},\left|\eta_{\mu_{2}}\right|<2.4$;

- $p_{\mathrm{T}}^{\mathrm{b} \text { jet }}>20 \mathrm{GeV}$ and $|\eta|<2.4$;

- $p_{\mathrm{T}}^{\text {miss }}<40 \mathrm{GeV}$.

The optimization of the selections was repeated for an A boson mass of $60 \mathrm{GeV}$ and similar values were obtained. The signal selection efficiency is 0.013 for $m_{\mathrm{A}}=30 \mathrm{GeV}$.

\section{$5 \quad$ Results and systematic uncertainties}

Figure 1 shows the transverse momentum of the leading (left) and subleading (right) muons, and figure 2 shows the $p_{\mathrm{T}}$ of the leading $\mathrm{b}$ jet (left) and the $p_{\mathrm{T}}^{\text {miss }}$ (right) for the events passing the selection requirements for the other variables. The histogram labeled as MC(Top) shows the sum of the single top and top quark pair production processes.

The upper limits on the signal contribution in the mass range of $25<m_{\mu \mu}<60 \mathrm{GeV}$ have been extracted using a fit of the binned background and signal templates to the dimuon mass distribution in the region $12<m_{\mu \mu}<70 \mathrm{GeV}$. The mass intervals of [12-25] and $[60-70] \mathrm{GeV}$ have been used for a verification of the background expectation obtained from the simulation.

The signal and background templates are obtained from simulation rescaled and corrected using information from data. To obtain signal mass shapes in $1 \mathrm{GeV}$ steps we perform a linear interpolation between histograms as a function of the mass using the algorithm described in ref. [49] and implemented in the Root package [50]. The $\mathrm{W}+$ jets and VV background event yields are found to be negligible. The predicted yields for the background processes take into account the following sources of systematic uncertainty, where the range of variations of each source was determined in dedicated studies with appropriate data control samples or, if relevant, from the theory uncertainty affecting the prediction:

- luminosity uncertainty, $2.6 \%$ [51]; 

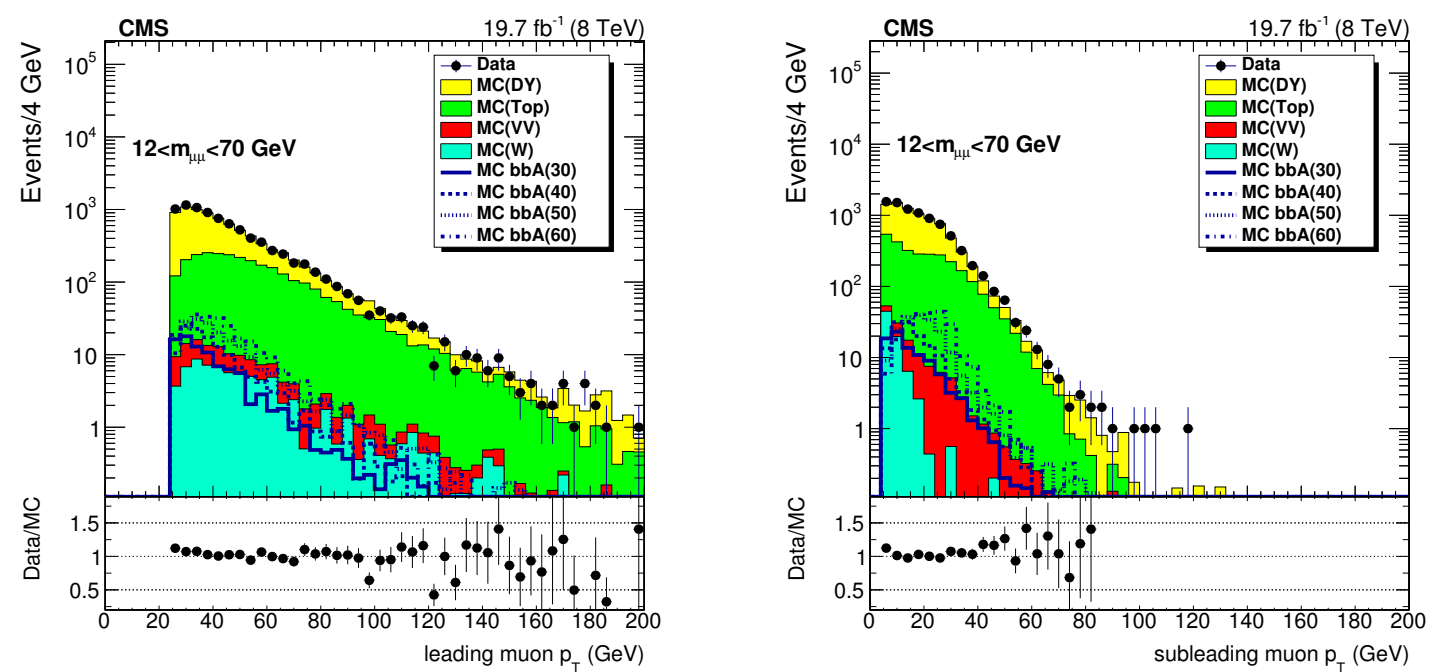

Figure 1. The transverse momentum of the leading (left) and the subleading (right) muon for data (dots) and simulation (histograms). The histograms for simulated backgrounds are stacked. The histogram labeled as MC(Top) shows the sum of the single top and top quark pair production processes. The expected signal is shown assuming a signal cross section times branching fraction of $350 \mathrm{fb}$. The background is normalized to the number of events expected from simulation. In the lower panel, the ratio of the number of events in data and background simulation is shown.
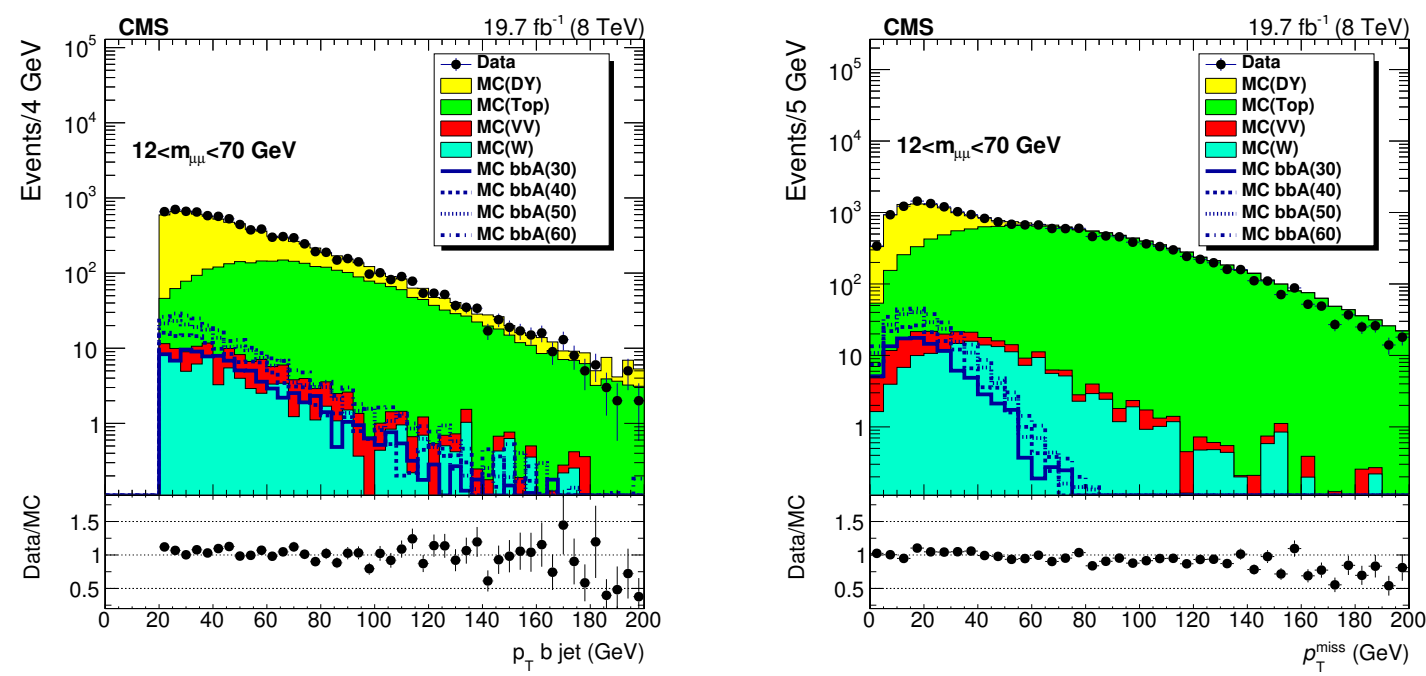

Figure 2. The transverse momentum of the leading $p_{\mathrm{T}} \mathrm{b}$ jet (left) and the missing transverse momentum (right) for data (dots) and simulation (histograms). The histograms for simulated backgrounds are stacked. The histogram labeled as MC(Top) shows the sum of the single top and top quark pair production processes. The expected signal is shown assuming a signal cross section times branching fraction of $350 \mathrm{fb}$. The background is normalized to the number of events expected from simulation. In the lower panel, the ratio of the number of events in data and background simulation is shown. 
- muon identification and isolation efficiency uncertainty, 3\%;

- top quark background normalization uncertainty, $7 \%$;

- jet energy scale uncertainty, $3 \%$ for the DY+jets and $0.2 \%$ for top quark backgrounds;

- jet energy resolution uncertainty, $0.3 \%$ for DY+jet and $0.1 \%$ for top quark backgrounds;

- pileup modeling uncertainty, $2 \%$ for DY+jets and $1 \%$ for top quark backgrounds;

- $E_{\mathrm{T}}^{\text {miss }}$ measurement uncertainty, $2 \%$ for DY+jets and $4 \%$ for top quark backgrounds.

While the above uncertainties only affect the expected total event yield, others can have an impact both on the total event yield and on the shape of the predicted $m_{\mu \mu}$ distribution. The following set of systematic uncertainties in the $m_{\mu \mu}$ background shape and the normalization have been taken into account:

- b tagging scaling factor uncertainty [47];

- renormalization and factorization scale, and PDF uncertainties;

The renormalization and factorization scale uncertainties are particularly important for the modeling of the shape of the DY+jets background, which after the final selections is dominated by the $\mu \mu \mathrm{b} \overline{\mathrm{b}}$ process. The scale uncertainty in the $\mu \mu \mathrm{b} \overline{\mathrm{b}}$ prediction, taken from simulation, has been evaluated as a function of the dimuon mass using the $\mathrm{pp} \rightarrow \mu \mu \mathrm{b} \overline{\mathrm{b}}$ process implemented in MADGRAPH5_aMC@NLO v2.3.0 in a four-flavor production scheme with massive b quarks [52]. The renormalization and factorization scales $\left(\mu_{\mathrm{R}}\right.$ and $\left.\mu_{\mathrm{F}}\right)$ have been varied simultaneously by factors of 0.5 and 2.0. The uncertainty on the differential cross-section as a function of the dimuon mass varies from 20 to $15 \%$ in the dimuon mass region of $12<m_{\mu \mu}<70 \mathrm{GeV}$. It is applied to the total DY+jets background, motivated by the fact the DY plus light-flavor jets background after the $\mathrm{b}$ tagging requirement is much smaller than the $\mu \mu \mathrm{b} \overline{\mathrm{b}}$ background, and the scale uncertainty for the $\mu \mu \mathrm{c} \overline{\mathrm{c}}$ process is expected to be similar to that for the $\mu \mu \mathrm{b} \overline{\mathrm{b}}$ process.

Uncertainties related to the imprecision in our knowledge of the PDFs are also evaluated. The uncertainty in the gluon-gluon luminosity is considered, since in the four-flavor scheme the dominant LO production process is gg $\rightarrow \mu \mu \mathrm{b} \overline{\mathrm{b}}$. The q $\overline{\mathrm{q}} \rightarrow \mu \mu \mathrm{b} \overline{\mathrm{b}}$ process represents only $\simeq 15 \%$ of the total cross-section. The gluon-gluon luminosity uncertainty is compared for three PDF sets, CT10NLO [53], MSTW2008NLO [54], and NNPDF2.3NLO [55]. In the mass region of $\simeq 30-70 \mathrm{GeV}$ the CT10NLO uncertainty envelope covers the uncertainties of the other two PDF sets, therefore the CT10NLO gluon-gluon luminosity uncertainty is used in the analysis. This PDF uncertainty is applied as a function of the dimuon mass to the total DY+jets background after $\mathrm{b}$ tagging has been applied. It is motivated by the fact that the $\mu \mu \mathrm{c} \overline{\mathrm{c}}$ and $\mu \mu \mathrm{b} \overline{\mathrm{b}}$ processes are both initiated by gluon-gluon interactions in the four-flavor scheme at LO.

The dimuon mass spectrum, the expected background, and its uncertainty are presented in figure 3 (left) together with the expected signal for $m_{\mathrm{A}}=30 \mathrm{GeV}$ assuming a 

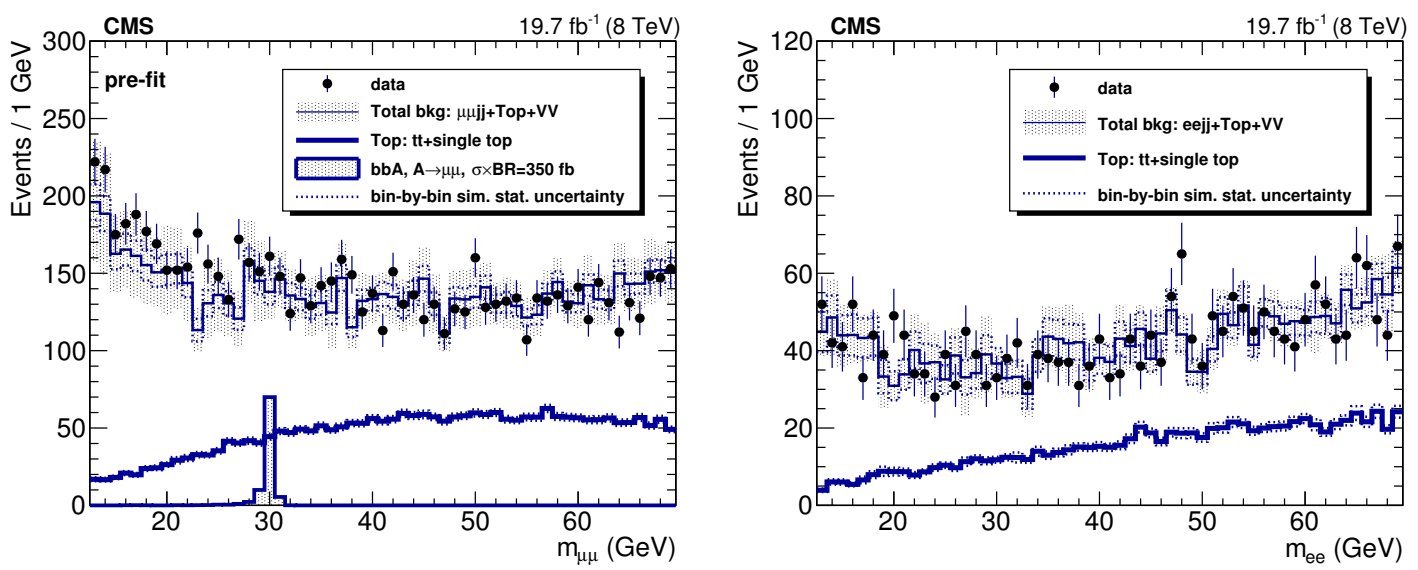

Figure 3. Left: the dimuon mass distribution with the pre-fit expected background event yield and its uncertainty, and the expected signal for $m_{\mathrm{A}}=30 \mathrm{GeV}$ assuming a signal cross section times branching fraction of $350 \mathrm{fb}$. Right: cross-check with the $\mathrm{e}^{+} \mathrm{e}^{-}$final state showing the dielectron mass spectrum with the expected background event yield and its uncertainty.

signal cross section times branching fraction of $350 \mathrm{fb}$. The $\mathrm{PF}$ jet reconstruction algorithm is used. There is a fair agreement between data and background.

To cross-check the background estimation technique that relies on the accuracy of the simulation for the background, we consider data where instead of requiring the presence of two muons, we require two opposite-sign electrons. Since the dielectron final state would be suppressed by $\left(m_{\mathrm{e}} / m_{\mu}\right)^{2}$ in the pseudoscalar Higgs decay, we do not consider it for our search. Kinematic thresholds and all other requirements are kept exactly the same as in the main analysis, except the threshold on the second leading $p_{\mathrm{T}}$ electron, which is taken higher than in the dimuon analysis $\left(p_{\mathrm{T}}^{\mathrm{e}_{2}}>10 \mathrm{GeV}\right)$ to reduce the QCD multijet background. The data are found to be in good agreement with the background model prediction, as illustrated in figure 3 (right).

To cross-check the effect of event migration on the exclusion limits due to the choice of jet reconstruction algorithm we use the JPT algorithm as an alternative and repeat the full analysis chain. Figure 4 shows the dimuon mass spectrum for events selected by both the main (PF) and alternative (JPT) jet reconstruction methods as well as for events selected by only the JPT algorithm. The two reconstruction methods select slightly different events. The fraction of migrated events due to the choice of jet algorithm is of the order of $20 \%$ and almost independent of the dimuon mass. The expected and observed upper limits obtained using PF or JPT jets are very similar. The final results presented use PF jets.

The experimental uncertainties considered above for the background are also taken into account for the signal. In addition, the uncertainty in the signal acceptance due to the signal modeling is evaluated using a MADGRAPH5_aMC@NLO+PYTHIA 8 particle level simulation of the $\mathrm{pp} \rightarrow \mathrm{b} \overline{\mathrm{b}} \mathrm{A}, \mathrm{A} \rightarrow \mu \mu$ process in the four-flavor production scheme [56-58]. It includes the uncertainty in the shower scale parameter, $\alpha$ (an upper scale allowed for the shower in PYTHIA) [13], the renormalization and factorization scales, and the PDFs. In the evaluation of the uncertainties we follow the recommendations of LHC Higgs Cross 


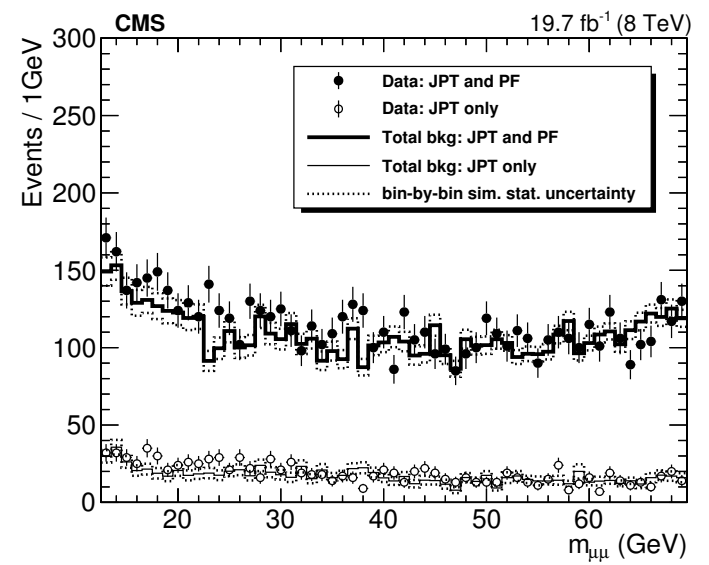

Figure 4. The dimuon mass distribution with the pre-fit expected background event yield for events selected by both the main (PF) and alternative (JPT) jet reconstruction methods as well as events selected by only the JPT algorithm.

section Working Group [59]. The renormalization and factorization scales are varied with two constraints such that $0.5 \mu_{0} \leq \mu_{\mathrm{R}}, \mu_{\mathrm{F}} \leq 2 \mu_{0}$ varies while requiring $0.5 \leq \mu_{\mathrm{R}} / \mu_{\mathrm{F}} \leq 2$ ( $\mu_{0}$ is the recommended central scale value [59]); the shower scale parameter is varied as $\alpha \in[1 /(4 \sqrt{2}), \sqrt{2} / 4]$. The PDF uncertainty is obtained with the PDF4LHC15_nlo_nf4_30 set [60-63]. The total theoretical uncertainty on the signal acceptance, taken as the linear sum of the shower scale, the renormalization and factorization scale, and the PDF uncertainties, varies from $-16 \%$ to $+18 \%$. The difference in the signal acceptance between the PYTHIA 6 and MADGRAPH5_aMC@NLO+PYTHIA 8 particle level simulation is less than the total theoretical uncertainty discussed above.

The dimuon mass distribution in figure 3 (left) is used in the evaluation of the limits on the cross section times branching fraction, $\sigma(\mathrm{pp} \rightarrow \mathrm{b} \overline{\mathrm{b}} \mathrm{A}) \mathcal{B}(\mathrm{A} \rightarrow \mu \mu)$. The limits have been extracted using a test statistic based on the profile likelihood ratio and asymptotic formulae, along with the constraint of a positive signal, as proposed in refs. [64, 65]. Systematic uncertainties are included in the form of nuisance parameters. The dimuon mass spectrum and the post-fit background event yield and its uncertainty given by the fit is presented in figure 5 (left), along with the expected signal for $m_{\mathrm{A}}=30 \mathrm{GeV}$ assuming a signal cross section times branching fraction of $350 \mathrm{fb}$.

We scan over A masses from 25 to $60 \mathrm{GeV}$. The expected and observed upper limits at $95 \%$ confidence level $(\mathrm{CL})$ on $\sigma(\mathrm{pp} \rightarrow \mathrm{b} \overline{\mathrm{b}} \mathrm{A}) \mathcal{B}(\mathrm{A} \rightarrow \mu \mu)$ are shown in figure 5 (right). Due to the good agreement between the data and the expected background before and after the fit the limits for all mass points in the mass interval of $25<m_{\mu \mu}<60 \mathrm{GeV}$ are within two standard deviations of the expected limit.

In the $2 \mathrm{HDM}$, the $\mathcal{B}(\mathrm{A} \rightarrow \mu \mu)$ and $\mathcal{B}(\mathrm{A} \rightarrow \tau \tau)$ are related through the ratio of the Yukawa couplings, and thus are proportional to the square of the lepton masses, i.e.

$$
\frac{\mathcal{B}(\mathrm{A} \rightarrow \tau \tau)}{\mathcal{B}(\mathrm{A} \rightarrow \mu \mu)}=\left(\frac{m_{\tau}}{m_{\mu}}\right)^{2}
$$

The open circles in figure 5 show the upper limits on $\sigma(\mathrm{pp} \rightarrow \mathrm{b} \overline{\mathrm{b}} \mathrm{A}) \mathcal{B}(\mathrm{A} \rightarrow \mu \mu)$ obtained from the limits on $\sigma(\mathrm{pp} \rightarrow \mathrm{b} \overline{\mathrm{b}} \mathrm{A}) \mathcal{B}(\mathrm{A} \rightarrow \tau \tau)$ evaluated in the CMS analysis of the $\mathrm{A} \rightarrow \tau \tau$ 

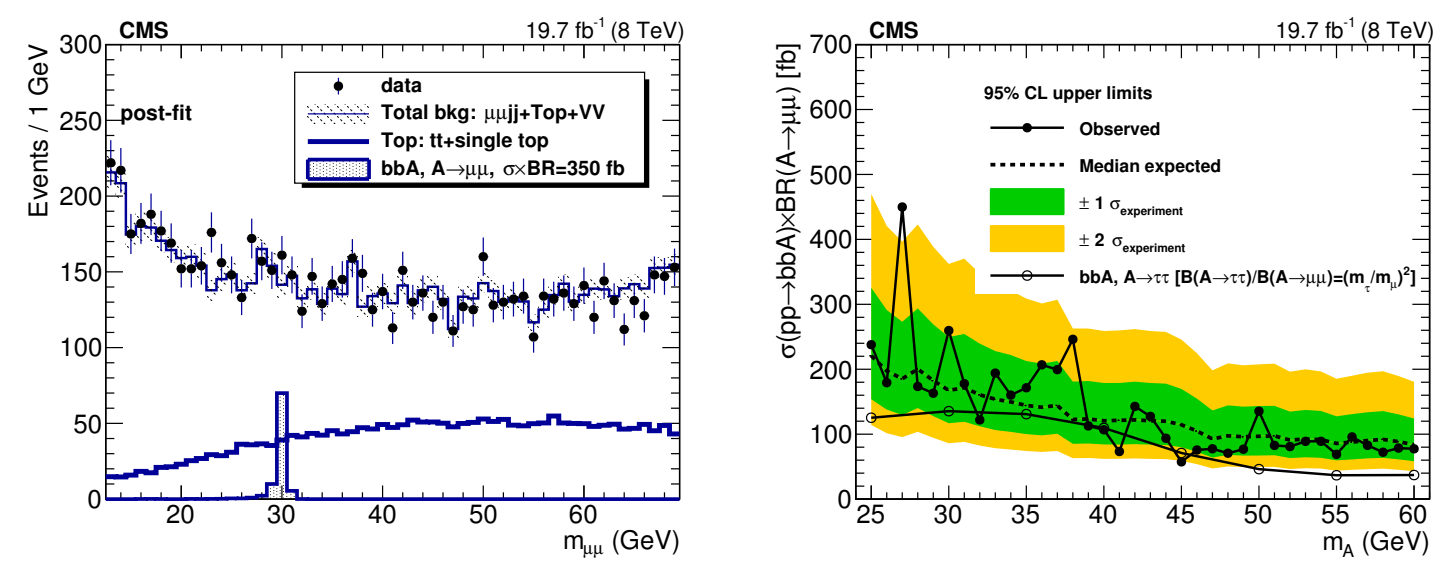

Figure 5. Left: the dimuon mass distribution with the post-fit background event yield and its uncertainty given by the fit, and the expected signal for $m_{\mathrm{A}}=30 \mathrm{GeV}$ assuming a signal cross section times branching fraction of $350 \mathrm{fb}$. Right: expected and observed upper limit at 95\% CL on $\sigma(\mathrm{pp} \rightarrow \mathrm{b} \overline{\mathrm{b}} \mathrm{A}) \mathcal{B A} \rightarrow \mu \mu)$ as a function of $m_{\mathrm{A}}$. The open circles show the limits obtained in the CMS analysis of the $\mathrm{A} \rightarrow \tau \tau$ final state [8] when translated into limits for the $\mathrm{A} \rightarrow \mu \mu$ final state using eq. (5.1).

final state [8]. One can see that upper limits evaluated from the direct search for the $\mathrm{A} \rightarrow \mu \mu$ decay in $\mathrm{b} \overline{\mathrm{b}} \mathrm{A}$ associated production are comparable with those from the $\mathrm{A} \rightarrow \tau \tau$ search using the same production process.

\section{Summary}

A light pseudoscalar Higgs boson, produced in association with a pair of $b$ jets and decaying into two muons, has been searched for in pp collisions at $\sqrt{s}=8 \mathrm{TeV}$ with an integrated luminosity of $19.7 \mathrm{fb}^{-1}$. This is the first time associated production with $\mathrm{b}$ quarks in the dimuon decay channel has been looked for in the low dimuon mass range. No signal has been observed in the dimuon mass range from 25 to $60 \mathrm{GeV}$. Upper limits on the cross section times branching fraction, $\sigma(\mathrm{pp} \rightarrow \mathrm{b} \overline{\mathrm{b}} \mathrm{A}) \mathcal{B}(\mathrm{A} \rightarrow \mu \mu)$, have been set. Despite the significantly lower branching fraction, the limits evaluated from the direct search for the $\mathrm{A} \rightarrow \mu \mu$ decay in $\mathrm{b} \overline{\mathrm{b}} \mathrm{A}$ associated production are comparable with those from the $\mathrm{A} \rightarrow \tau \tau$ search using the same production process.

\section{Acknowledgments}

We congratulate our colleagues in the CERN accelerator departments for the excellent performance of the LHC and thank the technical and administrative staffs at CERN and at other CMS institutes for their contributions to the success of the CMS effort. In addition, we gratefully acknowledge the computing centers and personnel of the Worldwide LHC Computing Grid for delivering so effectively the computing infrastructure essential to our analyses. Finally, we acknowledge the enduring support for the construction and operation of the LHC and the CMS detector provided by the following funding agencies: BMWFW and FWF (Austria); FNRS and FWO (Belgium); CNPq, CAPES, FAPERJ, 
and FAPESP (Brazil); MES (Bulgaria); CERN; CAS, MoST, and NSFC (China); COLCIENCIAS (Colombia); MSES and CSF (Croatia); RPF (Cyprus); SENESCYT (Ecuador); MoER, ERC IUT, and ERDF (Estonia); Academy of Finland, MEC, and HIP (Finland); CEA and CNRS/IN2P3 (France); BMBF, DFG, and HGF (Germany); GSRT (Greece); OTKA and NIH (Hungary); DAE and DST (India); IPM (Iran); SFI (Ireland); INFN (Italy); MSIP and NRF (Republic of Korea); LAS (Lithuania); MOE and UM (Malaysia); BUAP, CINVESTAV, CONACYT, LNS, SEP, and UASLP-FAI (Mexico); MBIE (New Zealand); PAEC (Pakistan); MSHE and NSC (Poland); FCT (Portugal); JINR (Dubna); MON, RosAtom, RAS, RFBR and RAEP (Russia); MESTD (Serbia); SEIDI, CPAN, PCTI and FEDER (Spain); Swiss Funding Agencies (Switzerland); MST (Taipei); ThEPCenter, IPST, STAR, and NSTDA (Thailand); TUBITAK and TAEK (Turkey); NASU and SFFR (Ukraine); STFC (United Kingdom); DOE and NSF (U.S.A.).

Individuals have received support from the Marie-Curie program and the European Research Council and Horizon 2020 Grant, contract No. 675440 (European Union); the Leventis Foundation; the A. P. Sloan Foundation; the Alexander von Humboldt Foundation; the Belgian Federal Science Policy Office; the Fonds pour la Formation à la Recherche dans l'Industrie et dans l'Agriculture (FRIA-Belgium); the Agentschap voor Innovatie door Wetenschap en Technologie (IWT-Belgium); the Ministry of Education, Youth and Sports (MEYS) of the Czech Republic; the Council of Science and Industrial Research, India; the HOMING PLUS program of the Foundation for Polish Science, cofinanced from European Union, Regional Development Fund, the Mobility Plus program of the Ministry of Science and Higher Education, the National Science Center (Poland), contracts Harmonia 2014/14/M/ST2/00428, Opus 2014/13/B/ST2/02543, 2014/15/B/ST2/03998, and 2015/19/B/ST2/02861, Sonata-bis 2012/07/E/ST2/01406; the National Priorities Research Program by Qatar National Research Fund; the Programa Clarín-COFUND del Principado de Asturias; the Thalis and Aristeia programs cofinanced by EU-ESF and the Greek NSRF; the Rachadapisek Sompot Fund for Postdoctoral Fellowship, Chulalongkorn University and the Chulalongkorn Academic into Its 2nd Century Project Advancement Project (Thailand); and the Welch Foundation, contract C-1845.

Open Access. This article is distributed under the terms of the Creative Commons Attribution License (CC-BY 4.0), which permits any use, distribution and reproduction in any medium, provided the original author(s) and source are credited.

\section{References}

[1] ATLAS collaboration, Observation of a new particle in the search for the standard model Higgs boson with the ATLAS detector at the LHC, Phys. Lett. B 716 (2012) 1 [arXiv: 1207.7214] [INSPIRE].

[2] CMS collaboration, Observation of a new boson at a mass of $125 \mathrm{GeV}$ with the CMS experiment at the LHC, Phys. Lett. B 716 (2012) 30 [arXiv:1207.7235] [INSPIRE].

[3] CMS collaboration, Observation of a new boson with mass near $125 \mathrm{GeV}$ in pp collisions at $\sqrt{s}=7$ and $8 \mathrm{TeV}$, JHEP 06 (2013) 081 [arXiv: 1303.4571] [INSPIRE]. 
[4] ATLAS and CMS collaborations, Combined measurement of the Higgs boson mass in pp collisions at $\sqrt{s}=7$ and 8 TeV with the ATLAS and CMS experiments, Phys. Rev. Lett. 114 (2015) 191803 [arXiv: 1503.07589] [INSPIRE].

[5] J.F. Gunion et al., The Higgs hunter's guide, Frontiers in Physics volume 80, Perseus Books, U.S.A. (2000).

[6] G.C. Branco et al., Theory and phenomenology of two-Higgs-doublet models, Phys. Rept. 516 (2012) 1 [arXiv: 1106.0034] [INSPIRE].

[7] J. Bernon, J.F. Gunion, Y. Jiang and S. Kraml, Light Higgs bosons in two-higgs-doublet models, Phys. Rev. D 91 (2015) 075019 [arXiv:1412.3385] [INSPIRE].

[8] CMS collaboration, Search for a low-mass pseudoscalar Higgs boson produced in association

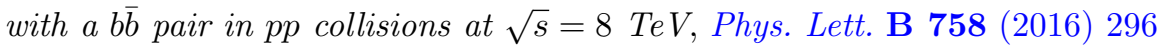
[arXiv: 1511.03610] [INSPIRE].

[9] CMS collaboration, Search for light bosons in decays of the $125 \mathrm{GeV}$ Higgs boson in proton-proton collisions at $\sqrt{s}=8 \mathrm{TeV}$, JHEP 10 (2017) 076 [arXiv: 1701.02032] [INSPIRE].

[10] CMS collaboration, The CMS experiment at the CERN LHC, 2008 JINST 3 S08004 [INSPIRE].

[11] T. Sjöstrand, S. Mrenna and P.Z. Skands, PYTHIA 6.4 physics and manual, JHEP 05 (2006) 026 [hep-ph/0603175] [INSPIRE].

[12] J. Alwall, M. Herquet, F. Maltoni, O. Mattelaer and T. Stelzer, MadGraph 5: going beyond, JHEP 06 (2011) 128 [arXiv: 1106.0522] [INSPIRE].

[13] J. Alwall et al., The automated computation of tree-level and next-to-leading order differential cross sections and their matching to parton shower simulations, JHEP 07 (2014) 079 [arXiv: 1405.0301] [INSPIRE].

[14] J. Pumplin, D.R. Stump, J. Huston, H.L. Lai, P.M. Nadolsky and W.K. Tung, New generation of parton distributions with uncertainties from global QCD analysis, JHEP 07 (2002) 012 [hep-ph/0201195] [INSPIRE].

[15] M.L. Mangano, M. Moretti, F. Piccinini and M. Treccani, Matching matrix elements and shower evolution for top-quark production in hadronic collisions, JHEP 01 (2007) 013 [hep-ph/0611129] [INSPIRE].

[16] J. Alwall et al., Comparative study of various algorithms for the merging of parton showers and matrix elements in hadronic collisions, Eur. Phys. J. C 53 (2008) 473 [arXiv:0706.2569] [INSPIRE].

[17] K. Melnikov and F. Petriello, Electroweak gauge boson production at hadron colliders through $O\left(\alpha_{s}^{2}\right)$, Phys. Rev. D 74 (2006) 114017 [hep-ph/0609070] [InSPIRE].

[18] M. Czakon, P. Fiedler and A. Mitov, Total top-quark pair-production cross section at hadron colliders through $O\left(\alpha_{S}^{4}\right)$, Phys. Rev. Lett. 110 (2013) 252004 [arXiv:1303.6254] [INSPIRE].

[19] S. Alioli, P. Nason, C. Oleari and E. Re, A general framework for implementing NLO calculations in shower Monte Carlo programs: the POWHEG BOX, JHEP 06 (2010) 043 [arXiv: 1002.2581] [INSPIRE].

[20] P. Nason, A new method for combining NLO QCD with shower Monte Carlo algorithms, JHEP 11 (2004) 040 [hep-ph/0409146] [INSPIRE]. 
[21] S. Frixione, P. Nason and C. Oleari, Matching NLO QCD computations with parton shower simulations: the POWHEG method, JHEP 11 (2007) 070 [arXiv: 0709. 2092] [INSPIRE].

[22] N. Kidonakis, Differential and total cross sections for top pair and single top productio, in the proceedings of the $20^{\text {th }}$ International Workshop on Deep-Inelastic Scattering and Related Subjects, May 26-30, Bonn, Germany (2012) [DESY-PROC-2012-02].

[23] N. Kidonakis, Top quark production, in the proceedings of the Helmholtz International Summer School on Physics of Heavy Quarks and Hadrons (HQ2013), July 15-28, JINR, Dubna, Russia (2014) [arXiv:1311.0283].

[24] T. Gehrmann et al., $W^{+} W^{-}$production at hadron colliders in next to next to leading order QCD, Phys. Rev. Lett. 113 (2014) 212001 [arXiv:1408.5243] [INSPIRE].

[25] J.M. Campbell and R.K. Ellis, MCFM for the Tevatron and the LHC, Nucl. Phys. Proc. Suppl. 205-206 (2010) 10 [arXiv: 1007.3492] [INSPIRE].

[26] CMS collaboration, Study of the underlying event at forward rapidity in pp collisions at $\sqrt{s}=0.9,2.76$ and $7 \mathrm{TeV}$, JHEP 04 (2013) 072 [arXiv:1302.2394] [INSPIRE].

[27] CMS collaboration, Event generator tunes obtained from underlying event and multiparton scattering measurements, Eur. Phys. J. C 76 (2016) 155 [arXiv:1512.00815] [inSPIRE].

[28] R. Field, Min-bias and the underlying event at the LHC, Acta Phys. Polon. B 42 (2011) 2631 [arXiv: 1110 . 5530] [INSPIRE].

[29] R. Field, Studying the 'underlying event' at CDF and the $L H C$, in the proceedings of the $1^{\text {st }}$ International Workshop on Multiple Partonic Interactions at the LHC (MPI08), October 27-31, Perugia, Italy (2009).

[30] GEANT4 collaboration J. Allison et al., GEANT4 developments and applications, IEEE Trans. Nucl. Sci. 53 (2006) 270 [INSPIRE].

[31] GEANT4 collaboration, S. Agostinelli et al., GEANT4 - a simulation toolkit, Nucl. Instrum. Meth. A 506 (2003) 250 [INSPIRE].

[32] CMS collaboration, Determination of jet energy calibration and transverse momentum resolution in CMS, 2011 JINST 6 P11002 [arXiv:1107.4277] [INSPIRE].

[33] CMS collaboration, Identification of b-quark jets with the CMS experiment, 2013 JINST 8 P04013 [arXiv: 1211.4462] [INSPIRE].

[34] CMS collaboration, Performance of CMS muon reconstruction in pp collision events at $\sqrt{s}=7 \mathrm{TeV}, 2012$ JINST 7 P10002 [arXiv:1206.4071] [INSPIRE].

[35] CMS collaboration, Performance of electron reconstruction and selection with the CMS detector in proton-proton collisions at $\sqrt{s}=8 \mathrm{TeV}, 2015$ JINST $10 \mathrm{P} 06005$ [arXiv: 1502.02701] [INSPIRE].

[36] M. Cacciari, G.P. Salam and G. Soyez, The anti-k $k_{t}$ jet clustering algorithm, JHEP 04 (2008) 063 [arXiv: 0802.1189] [INSPIRE].

[37] M. Cacciari, G.P. Salam and G. Soyez, FastJet user manual, Eur. Phys. J. C 72 (2012) 1896 [arXiv:1111.6097] [INSPIRE].

[38] CMS collaboration, Performance of jet-plus-tracks algorithm in run I, CMS-PAS-JME-14-005 (2014).

[39] CMS collaboration, Particle-flow reconstruction and global event description with the CMS detector, 2017 JINST 12 P10003 [arXiv:1706. 04965] [INSPIRE]. 
[40] CMS collaboration, Pileup Jet Identification, CMS-PAS-JME-13-005 (2013).

[41] M. Cacciari and G.P. Salam, Pileup subtraction using jet areas, Phys. Lett. B 659 (2008) 119 [arXiv: 0707.1378] [INSPIRE].

[42] M. Cacciari, G.P. Salam and G. Soyez, The catchment area of jets, JHEP 04 (2008) 005 [arXiv: 0802.1188] [INSPIRE].

[43] CMS collaboration, Missing transverse energy performance of the CMS detector, 2011 JINST 6 P09001 [arXiv:1106.5048] [INSPIRE].

[44] CMS collaboration, Identification and filtering of uncharacteristic noise in the CMS hadron calorimeter, 2010 JINST 5 T03014 [arXiv:0911.4881] [INSPIRE].

[45] CMS collaboration, Jet energy scale and resolution in the CMS experiment in pp collisions at $8 \mathrm{TeV}, 2017$ JINST 12 P02014 [arXiv: 1607.03663] [INSPIRE].

[46] A. Hocker et al., TMVA - Toolkit for multivariate data analysis, PoS (ACAT) 040 [physics/0703039] [INSPIRE].

[47] CMS Collaboration, Performance of b tagging at $\sqrt{s}=8 \mathrm{TeV}$ in multijet, ttbar and boosted topology events, CMS-PAS-BTV-13-001 (2013).

[48] S.I. Bityukov and N.V. Krasnikov, New physics discovery potential in future experiments, Mod. Phys. Lett. A 13 (1998) 3235 [physics/9811025] [InSPIRE].

[49] A.L. Read, Linear interpolation of histograms, Nucl. Instrum. Meth. A 425 (1999) 357 [INSPIRE].

[50] R. Brun and F. Rademakers, ROOT: an object oriented data analysis framework, Nucl. Instrum. Meth. A 389 (1997) 81 [inSPIRE].

[51] CMS collaboration, CMS luminosity based on pixel cluster counting - Summer 2013 Update, CMS-PAS-LUM-13-001 (2013).

[52] R. Frederix, S. Frixione, V. Hirschi, F. Maltoni, R. Pittau and P. Torrielli, $W$ and $Z / \gamma *$ boson production in association with a bottom-antibottom pair, JHEP 09 (2011) 061 [arXiv:1106.6019] [INSPIRE].

[53] H.-L. Lai et al., New parton distributions for collider physics, Phys. Rev. D 82 (2010) 074024 [arXiv: 1007.2241] [INSPIRE].

[54] A.D. Martin, W.J. Stirling, R.S. Thorne and G. Watt, Heavy-quark mass dependence in global PDF analyses and 3- and 4-flavour parton distributions, Eur. Phys. J. C 70 (2010) 51 [arXiv: 1007.2624] [INSPIRE].

[55] NNPDF collaboration, R.D. Ball et al., Parton distributions with LHC data, Nucl. Phys. B 867 (2013) 244 [arXiv:1207.1303] [INSPIRE].

[56] M. Wiesemann et al., Higgs production in association with bottom quarks, JHEP 02 (2015) 132 [arXiv: 1409.5301] [INSPIRE].

[57] S. Dittmaier, M. Krämer and M. Spira, Higgs radiation off bottom quarks at the Tevatron and the CERN LHC, Phys. Rev. D 70 (2004) 074010 [hep-ph/0309204] [INSPIRE].

[58] S. Dawson, C.B. Jackson, L. Reina and D. Wackeroth, Exclusive Higgs boson production with bottom quarks at hadron colliders, Phys. Rev. D 69 (2004) 074027 [hep-ph/0311067] [INSPIRE]. 
[59] LHC Higgs Cross Section Working Group collaboration, D. de Florian et al., Handbook of LHC Higgs cross sections: 4. Deciphering the nature of the Higgs sector, arXiv: 1610.07922 [INSPIRE].

[60] J. Butterworth et al., PDF4LHC recommendations for LHC Run II, J. Phys. G 43 (2016) 023001 [arXiv: 1510.03865 ] [INSPIRE].

[61] NNPDF collaboration, R.D. Ball et al., Parton distributions for the LHC Run II, JHEP 04 (2015) 040 [arXiv: 1410.8849] [INSPIRE].

[62] S. Dulat et al., New parton distribution functions from a global analysis of quantum chromodynamics, Phys. Rev. D 93 (2016) 033006 [arXiv: 1506.07443] [InSPIRE].

[63] L.A. Harland-Lang, A.D. Martin, P. Motylinski and R.S. Thorne, Parton distributions in the LHC era: MMHT 2014 PDFs, Eur. Phys. J. C 75 (2015) 204 [arXiv:1412.3989] [InSPIRE].

[64] G. Cowan, K. Cranmer, E. Gross and O. Vitells, Asymptotic formulae for likelihood-based tests of new physics, Eur. Phys. J. C 71 (2011) 1554 [Erratum ibid. C 73 (2013) 2501] [arXiv: 1007.1727] [INSPIRE].

[65] ATLAS, CMS collaborations and LHC Higgs Combination Group, Procedure for the LHC Higgs boson search combination in Summer 2011, CMS-NOTE-2011-005 (2011). 


\section{The CMS collaboration}

\section{Yerevan Physics Institute, Yerevan, Armenia}

A.M. Sirunyan, A. Tumasyan

\section{Institut für Hochenergiephysik, Wien, Austria}

W. Adam, F. Ambrogi, E. Asilar, T. Bergauer, J. Brandstetter, E. Brondolin, M. Dragicevic, J. Erö, M. Flechl, M. Friedl, R. Frühwirth ${ }^{1}$, V.M. Ghete, J. Grossmann, J. Hrubec, M. Jeitler ${ }^{1}$, A. König, N. Krammer, I. Krätschmer, D. Liko, T. Madlener, I. Mikulec, E. Pree, D. Rabady, N. Rad, H. Rohringer, J. Schieck ${ }^{1}$, R. Schöfbeck, M. Spanring, D. Spitzbart, W. Waltenberger, J. Wittmann, C.-E. Wulz ${ }^{1}$, M. Zarucki

Institute for Nuclear Problems, Minsk, Belarus

V. Chekhovsky, V. Mossolov, J. Suarez Gonzalez

\section{Universiteit Antwerpen, Antwerpen, Belgium}

E.A. De Wolf, D. Di Croce, X. Janssen, J. Lauwers, H. Van Haevermaet, P. Van Mechelen, N. Van Remortel

\section{Vrije Universiteit Brussel, Brussel, Belgium}

S. Abu Zeid, F. Blekman, J. D’Hondt, I. De Bruyn, J. De Clercq, K. Deroover, G. Flouris, D. Lontkovskyi, S. Lowette, S. Moortgat, L. Moreels, Q. Python, K. Skovpen, S. Tavernier, W. Van Doninck, P. Van Mulders, I. Van Parijs

\section{Université Libre de Bruxelles, Bruxelles, Belgium}

H. Brun, B. Clerbaux, G. De Lentdecker, H. Delannoy, G. Fasanella, L. Favart, R. Goldouzian, A. Grebenyuk, G. Karapostoli, T. Lenzi, J. Luetic, T. Maerschalk, A. Marinov, A. Randle-conde, T. Seva, C. Vander Velde, P. Vanlaer, D. Vannerom, R. Yonamine, F. Zenoni, F. Zhang ${ }^{2}$

\section{Ghent University, Ghent, Belgium}

A. Cimmino, T. Cornelis, D. Dobur, A. Fagot, M. Gul, I. Khvastunov, D. Poyraz, C. Roskas, S. Salva, M. Tytgat, W. Verbeke, N. Zaganidis

\section{Université Catholique de Louvain, Louvain-la-Neuve, Belgium}

H. Bakhshiansohi, O. Bondu, S. Brochet, G. Bruno, A. Caudron, S. De Visscher, C. Delaere, M. Delcourt, B. Francois, A. Giammanco, A. Jafari, M. Komm, G. Krintiras, V. Lemaitre, A. Magitteri, A. Mertens, M. Musich, K. Piotrzkowski, L. Quertenmont, M. Vidal Marono, S. Wertz

\section{Université de Mons, Mons, Belgium}

N. Beliy

\section{Centro Brasileiro de Pesquisas Fisicas, Rio de Janeiro, Brazil}

W.L. Aldá Júnior, F.L. Alves, G.A. Alves, L. Brito, M. Correa Martins Junior, C. Hensel, A. Moraes, M.E. Pol, P. Rebello Teles 
Universidade do Estado do Rio de Janeiro, Rio de Janeiro, Brazil

E. Belchior Batista Das Chagas, W. Carvalho, J. Chinellato ${ }^{3}$, A. Custódio, E.M. Da Costa, G.G. Da Silveira ${ }^{4}$, D. De Jesus Damiao, S. Fonseca De Souza, L.M. Huertas Guativa, H. Malbouisson, M. Melo De Almeida, C. Mora Herrera, L. Mundim, H. Nogima, A. Santoro, A. Sznajder, E.J. Tonelli Manganote ${ }^{3}$, F. Torres Da Silva De Araujo, A. Vilela Pereira

Universidade Estadual Paulista $^{a}$, Universidade Federal do ABC ${ }^{b}$, São Paulo, Brazil

S. Ahuja ${ }^{a}$, C.A. Bernardes ${ }^{a}$, T.R. Fernandez Perez Tomei ${ }^{a}$, E.M. Gregores ${ }^{b}$, P.G. Mercadante ${ }^{b}$, S.F. Novaes ${ }^{a}$, Sandra S. Padula ${ }^{a}$, D. Romero $\operatorname{Abad}^{b}$, J.C. Ruiz $\operatorname{Vargas}^{a}$

Institute for Nuclear Research and Nuclear Energy of Bulgaria Academy of Sciences

A. Aleksandrov, R. Hadjiiska, P. Iaydjiev, M. Misheva, M. Rodozov, M. Shopova, S. Stoykova, G. Sultanov

University of Sofia, Sofia, Bulgaria

A. Dimitrov, I. Glushkov, L. Litov, B. Pavlov, P. Petkov

Beihang University, Beijing, China

W. Fang ${ }^{5}$, X. Gao ${ }^{5}$

Institute of High Energy Physics, Beijing, China

M. Ahmad, J.G. Bian, G.M. Chen, H.S. Chen, M. Chen, Y. Chen, C.H. Jiang, D. Leggat, H. Liao, Z. Liu, F. Romeo, S.M. Shaheen, A. Spiezia, J. Tao, C. Wang, Z. Wang, E. Yazgan, H. Zhang, J. Zhao

State Key Laboratory of Nuclear Physics and Technology, Peking University, Beijing, China

Y. Ban, G. Chen, Q. Li, S. Liu, Y. Mao, S.J. Qian, D. Wang, Z. Xu

Universidad de Los Andes, Bogota, Colombia

C. Avila, A. Cabrera, L.F. Chaparro Sierra, C. Florez, C.F. González Hernández, J.D. Ruiz Alvarez

University of Split, Faculty of Electrical Engineering, Mechanical Engineering and Naval Architecture, Split, Croatia

B. Courbon, N. Godinovic, D. Lelas, I. Puljak, P.M. Ribeiro Cipriano, T. Sculac

University of Split, Faculty of Science, Split, Croatia

Z. Antunovic, M. Kovac

Institute Rudjer Boskovic, Zagreb, Croatia

V. Brigljevic, D. Ferencek, K. Kadija, B. Mesic, A. Starodumov ${ }^{6}$, T. Susa

University of Cyprus, Nicosia, Cyprus

M.W. Ather, A. Attikis, G. Mavromanolakis, J. Mousa, C. Nicolaou, F. Ptochos, P.A. Razis, H. Rykaczewski 
Charles University, Prague, Czech Republic

M. Finger ${ }^{7}$, M. Finger Jr. ${ }^{7}$

Universidad San Francisco de Quito, Quito, Ecuador

E. Carrera Jarrin

Academy of Scientific Research and Technology of the Arab Republic of Egypt, Egyptian Network of High Energy Physics, Cairo, Egypt

Y. Assran $^{8,9}$, S. Elgammal ${ }^{9}$, A. Mahrous ${ }^{10}$

National Institute of Chemical Physics and Biophysics, Tallinn, Estonia

R.K. Dewanjee, M. Kadastik, L. Perrini, M. Raidal, A. Tiko, C. Veelken

Department of Physics, University of Helsinki, Helsinki, Finland

P. Eerola, J. Pekkanen, M. Voutilainen

Helsinki Institute of Physics, Helsinki, Finland

J. Härkönen, T. Järvinen, V. Karimäki, R. Kinnunen, T. Lampén, K. Lassila-Perini,

S. Lehti, T. Lindén, P. Luukka, E. Tuominen, J. Tuominiemi, E. Tuovinen

Lappeenranta University of Technology, Lappeenranta, Finland

J. Talvitie, T. Tuuva

IRFU, CEA, Université Paris-Saclay, Gif-sur-Yvette, France

M. Besancon, F. Couderc, M. Dejardin, D. Denegri, J.L. Faure, F. Ferri, S. Ganjour, S. Ghosh, A. Givernaud, P. Gras, G. Hamel de Monchenault, P. Jarry, I. Kucher, E. Locci, M. Machet, J. Malcles, G. Negro, J. Rander, A. Rosowsky, M.Ö. Sahin, M. Titov

Laboratoire Leprince-Ringuet, Ecole polytechnique, CNRS/IN2P3, Université Paris-Saclay, Palaiseau, France

A. Abdulsalam, I. Antropov, S. Baffioni, F. Beaudette, P. Busson, L. Cadamuro, C. Charlot, R. Granier de Cassagnac, M. Jo, S. Lisniak, A. Lobanov, J. Martin Blanco, M. Nguyen, C. Ochando, G. Ortona, P. Paganini, P. Pigard, S. Regnard, R. Salerno, J.B. Sauvan, Y. Sirois, A.G. Stahl Leiton, T. Strebler, Y. Yilmaz, A. Zabi, A. Zghiche

Université de Strasbourg, CNRS, IPHC UMR 7178, F-67000 Strasbourg, France

J.-L. Agram ${ }^{11}$, J. Andrea, D. Bloch, J.-M. Brom, M. Buttignol, E.C. Chabert, N. Chanon, C. Collard, E. Conte ${ }^{11}$, X. Coubez, J.-C. Fontaine ${ }^{11}$, D. Gelé, U. Goerlach, M. Jansová, A.-C. Le Bihan, N. Tonon, P. Van Hove

Centre de Calcul de l'Institut National de Physique Nucleaire et de Physique des Particules, CNRS/IN2P3, Villeurbanne, France

S. Gadrat

Université de Lyon, Université Claude Bernard Lyon 1, CNRS-IN2P3, Institut de Physique Nucléaire de Lyon, Villeurbanne, France

S. Beauceron, C. Bernet, G. Boudoul, R. Chierici, D. Contardo, P. Depasse, H. El Mamouni, J. Fay, L. Finco, S. Gascon, M. Gouzevitch, G. Grenier, B. Ille, F. Lagarde, I.B. Laktineh, 
M. Lethuillier, L. Mirabito, A.L. Pequegnot, S. Perries, A. Popov ${ }^{12}$, V. Sordini, M. Vander Donckt, S. Viret

Georgian Technical University, Tbilisi, Georgia

T. Toriashvili ${ }^{13}$

Tbilisi State University, Tbilisi, Georgia

Z. Tsamalaidze ${ }^{7}$

RWTH Aachen University, I. Physikalisches Institut, Aachen, Germany

C. Autermann, S. Beranek, L. Feld, M.K. Kiesel, K. Klein, M. Lipinski, M. Preuten,

C. Schomakers, J. Schulz, T. Verlage

RWTH Aachen University, III. Physikalisches Institut A, Aachen, Germany

A. Albert, E. Dietz-Laursonn, D. Duchardt, M. Endres, M. Erdmann, S. Erdweg, T. Esch,

R. Fischer, A. Güth, M. Hamer, T. Hebbeker, C. Heidemann, K. Hoepfner, S. Knutzen,

M. Merschmeyer, A. Meyer, P. Millet, S. Mukherjee, M. Olschewski, K. Padeken, T. Pook,

M. Radziej, H. Reithler, M. Rieger, F. Scheuch, D. Teyssier, S. Thüer

RWTH Aachen University, III. Physikalisches Institut B, Aachen, Germany

G. Flügge, B. Kargoll, T. Kress, A. Künsken, J. Lingemann, T. Müller, A. Nehrkorn, A. Nowack, C. Pistone, O. Pooth, A. Stahl ${ }^{14}$

\section{Deutsches Elektronen-Synchrotron, Hamburg, Germany}

M. Aldaya Martin, T. Arndt, C. Asawatangtrakuldee, K. Beernaert, O. Behnke, U. Behrens, A. Bermúdez Martínez, A.A. Bin Anuar, K. Borras ${ }^{15}$, V. Botta, A. Campbell, P. Connor, C. Contreras-Campana, F. Costanza, C. Diez Pardos, G. Eckerlin, D. Eckstein, T. Eichhorn, E. Eren, E. Gallo ${ }^{16}$, J. Garay Garcia, A. Geiser, A. Gizhko, J.M. Grados Luyando, A. Grohsjean, P. Gunnellini, M. Guthoff, A. Harb, J. Hauk, M. Hempel ${ }^{17}$, H. Jung, A. Kalogeropoulos, M. Kasemann, J. Keaveney, C. Kleinwort, I. Korol, D. Krücker, W. Lange, A. Lelek, T. Lenz, J. Leonard, K. Lipka, W. Lohmann ${ }^{17}$, R. Mankel, I.-A. Melzer-Pellmann, A.B. Meyer, G. Mittag, J. Mnich, A. Mussgiller, E. Ntomari, D. Pitzl, A. Raspereza, B. Roland, M. Savitskyi, P. Saxena, R. Shevchenko, S. Spannagel, N. Stefaniuk, G.P. Van Onsem, R. Walsh, Y. Wen, K. Wichmann, C. Wissing, O. Zenaiev

\section{University of Hamburg, Hamburg, Germany}

S. Bein, V. Blobel, M. Centis Vignali, T. Dreyer, E. Garutti, D. Gonzalez, J. Haller, A. Hinzmann, M. Hoffmann, A. Karavdina, R. Klanner, R. Kogler, N. Kovalchuk, S. Kurz, T. Lapsien, I. Marchesini, D. Marconi, M. Meyer, M. Niedziela, D. Nowatschin, F. Pantaleo ${ }^{14}$, T. Peiffer, A. Perieanu, C. Scharf, P. Schleper, A. Schmidt, S. Schumann, J. Schwandt, J. Sonneveld, H. Stadie, G. Steinbrück, F.M. Stober, M. Stöver, H. Tholen, D. Troendle, E. Usai, L. Vanelderen, A. Vanhoefer, B. Vormwald

Institut für Experimentelle Kernphysik, Karlsruhe, Germany

M. Akbiyik, C. Barth, S. Baur, E. Butz, R. Caspart, T. Chwalek, F. Colombo, W. De Boer, A. Dierlamm, B. Freund, R. Friese, M. Giffels, A. Gilbert, D. Haitz, F. Hartmann ${ }^{14}$, 
S.M. Heindl, U. Husemann, F. Kassel ${ }^{14}$, S. Kudella, H. Mildner, M.U. Mozer, Th. Müller, M. Plagge, G. Quast, K. Rabbertz, M. Schröder, I. Shvetsov, G. Sieber, H.J. Simonis,

R. Ulrich, S. Wayand, M. Weber, T. Weiler, S. Williamson, C. Wöhrmann, R. Wolf

Institute of Nuclear and Particle Physics (INPP), NCSR Demokritos, Aghia Paraskevi, Greece

G. Anagnostou, G. Daskalakis, T. Geralis, V.A. Giakoumopoulou, A. Kyriakis, D. Loukas, I. Topsis-Giotis

National and Kapodistrian University of Athens, Athens, Greece

G. Karathanasis, S. Kesisoglou, A. Panagiotou, N. Saoulidou

University of Ioánnina, Ioánnina, Greece

I. Evangelou, C. Foudas, P. Kokkas, S. Mallios, N. Manthos, I. Papadopoulos, E. Paradas, J. Strologas, F.A. Triantis

MTA-ELTE Lendület CMS Particle and Nuclear Physics Group, Eötvös Loránd University, Budapest, Hungary

M. Csanad, N. Filipovic, G. Pasztor, G.I. Veres ${ }^{18}$

Wigner Research Centre for Physics, Budapest, Hungary

G. Bencze, C. Hajdu, D. Horvath ${ }^{19}$, Á. Hunyadi, F. Sikler, V. Veszpremi, G. Vesztergombi ${ }^{18}$, A.J. Zsigmond

Institute of Nuclear Research ATOMKI, Debrecen, Hungary

N. Beni, S. Czellar, J. Karancsi ${ }^{20}$, A. Makovec, J. Molnar, Z. Szillasi

Institute of Physics, University of Debrecen, Debrecen, Hungary

M. Bartók ${ }^{18}$, P. Raics, Z.L. Trocsanyi, B. Ujvari

Indian Institute of Science (IISc), Bangalore, India

S. Choudhury, J.R. Komaragiri

National Institute of Science Education and Research, Bhubaneswar, India

S. Bahinipati ${ }^{21}$, S. Bhowmik, P. Mal, K. Mandal, A. Nayak ${ }^{22}$, D.K. Sahoo ${ }^{21}$, N. Sahoo, S.K. Swain

Panjab University, Chandigarh, India

S. Bansal, S.B. Beri, V. Bhatnagar, R. Chawla, N. Dhingra, A.K. Kalsi, A. Kaur, M. Kaur, R. Kumar, P. Kumari, A. Mehta, J.B. Singh, G. Walia

University of Delhi, Delhi, India

Ashok Kumar, Aashaq Shah, A. Bhardwaj, S. Chauhan, B.C. Choudhary, R.B. Garg, S. Keshri, A. Kumar, S. Malhotra, M. Naimuddin, K. Ranjan, R. Sharma, V. Sharma

Saha Institute of Nuclear Physics, HBNI, Kolkata, India

R. Bhardwaj, R. Bhattacharya, S. Bhattacharya, U. Bhawandeep, S. Dey, S. Dutt, S. Dutta,

S. Ghosh, N. Majumdar, A. Modak, K. Mondal, S. Mukhopadhyay, S. Nandan, A. Purohit, A. Roy, D. Roy, S. Roy Chowdhury, S. Sarkar, M. Sharan, S. Thakur 
Indian Institute of Technology Madras, Madras, India

P.K. Behera

Bhabha Atomic Research Centre, Mumbai, India

R. Chudasama, D. Dutta, V. Jha, V. Kumar, A.K. Mohanty ${ }^{14}$, P.K. Netrakanti, L.M. Pant, P. Shukla, A. Topkar

Tata Institute of Fundamental Research-A, Mumbai, India

T. Aziz, S. Dugad, B. Mahakud, S. Mitra, G.B. Mohanty, N. Sur, B. Sutar

Tata Institute of Fundamental Research-B, Mumbai, India

S. Banerjee, S. Bhattacharya, S. Chatterjee, P. Das, M. Guchait, Sa. Jain, S. Kumar, M. Maity ${ }^{23}$, G. Majumder, K. Mazumdar, T. Sarkar ${ }^{23}$, N. Wickramage ${ }^{24}$

Indian Institute of Science Education and Research (IISER), Pune, India

S. Chauhan, S. Dube, V. Hegde, A. Kapoor, K. Kothekar, S. Pandey, A. Rane, S. Sharma

Institute for Research in Fundamental Sciences (IPM), Tehran, Iran

S. Chenarani ${ }^{25}$, E. Eskandari Tadavani, S.M. Etesami ${ }^{25}$, M. Khakzad, M. Mohammadi Najafabadi, M. Naseri, S. Paktinat Mehdiabadi ${ }^{26}$, F. Rezaei Hosseinabadi, B. Safarzadeh ${ }^{27}$, M. Zeinali

\section{University College Dublin, Dublin, Ireland}

M. Felcini, M. Grunewald

INFN Sezione di Bari ${ }^{a}$, Università di Bari ${ }^{b}$, Politecnico di Bari ${ }^{c}$, Bari, Italy M. Abbrescia ${ }^{a, b}$, C. Calabria ${ }^{a, b}$, C. Caputo ${ }^{a, b}$, A. Colaleo $^{a}$, D. Creanza ${ }^{a, c}$, L. Cristella $^{a, b}$, N. De Filippis ${ }^{a, c}$, M. De Palma ${ }^{a, b}$, F. Errico ${ }^{a, b}$, L. Fiore ${ }^{a}$, G. Iasellia ${ }^{a, c}$, S. Lezki $^{a, b}$,

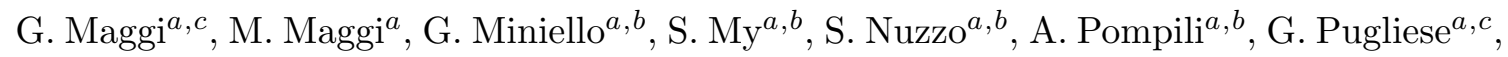
R. Radogna ${ }^{a, b}$, A. Ranieri ${ }^{a}$, G. Selvaggi ${ }^{a}, b$, A. Sharma ${ }^{a}$, L. Silvestris ${ }^{a, 14}$, R. Venditti ${ }^{a}$, P. Verwilligen ${ }^{a}$

INFN Sezione di Bologna ${ }^{a}$, Università di Bologna ${ }^{b}$, Bologna, Italy

G. Abbiendi ${ }^{a}$, C. Battilana ${ }^{a, b}$, D. Bonacorsi ${ }^{a}, b$, S. Braibant-Giacomelli ${ }^{a}, b$, R. Campanini $^{a, b}{ }$, P. Capiluppi ${ }^{a, b}$, A. Castro ${ }^{a, b}$, F.R. Cavallo ${ }^{a}$, S.S. Chhibra ${ }^{a}$, G. Codispoti ${ }^{a, b}$, M. Cuffiani ${ }^{a, b}$, G.M. Dallavalle ${ }^{a}$, F. Fabbri ${ }^{a}$, A. Fanfani ${ }^{a}, b$, D. Fasanella ${ }^{a, b}$, P. Giacomelli ${ }^{a}$, C. Grandi ${ }^{a}$, L. Guiducci ${ }^{a}, b$, S. Marcellini ${ }^{a}$, G. Masetti ${ }^{a}$, A. Montanari ${ }^{a}$, F.L. Navarria ${ }^{a, b}$, A. Perrotta ${ }^{a}$, A.M. Rossi ${ }^{a, b}$, T. Rovelli ${ }^{a, b}$, G.P. Siroli ${ }^{a, b}$, N. Tosi ${ }^{a}$

INFN Sezione di Catania ${ }^{a}$, Università di Catania ${ }^{b}$, Catania, Italy

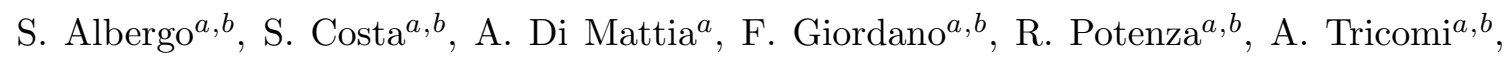
C. Tuve ${ }^{a, b}$

INFN Sezione di Firenze ${ }^{a}$, Università di Firenze ${ }^{b}$, Firenze, Italy

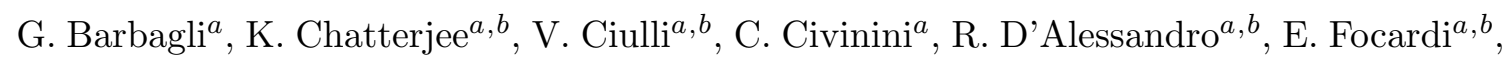

P. Lenzi ${ }^{a, b}$, M. Meschini ${ }^{a}$, S. Paoletti ${ }^{a}$, L. Russo ${ }^{a, 28}$, G. Sguazzoni ${ }^{a}$, D. Strom $^{a}$,

L. Viliani ${ }^{a, b, 14}$ 
INFN Laboratori Nazionali di Frascati, Frascati, Italy

L. Benussi, S. Bianco, F. Fabbri, D. Piccolo, F. Primavera ${ }^{14}$

INFN Sezione di Genova ${ }^{a}$, Università di Genova ${ }^{b}$, Genova, Italy

V. Calvelli ${ }^{a, b}$, F. Ferro ${ }^{a}$, E. Robutti $^{a}$, S. Tosi $^{a, b}$

INFN Sezione di Milano-Bicocca ${ }^{a}$, Università di Milano-Bicocca ${ }^{b}$, Milano, Italy

L. Brianza ${ }^{a, b}$, F. Brivio ${ }^{a, b}$, V. Ciriolo ${ }^{a, b}$, M.E. Dinardo ${ }^{a, b}$, S. Fiorendi ${ }^{a, b}$, S. Gennai $^{a}$,

A. Ghezzi ${ }^{a, b}$, P. Govoni ${ }^{a}, b$, S. Gundacker, M. Malberti ${ }^{a, b}$, S. Malvezzi ${ }^{a}$, R.A. Manzoni ${ }^{a, b}$,

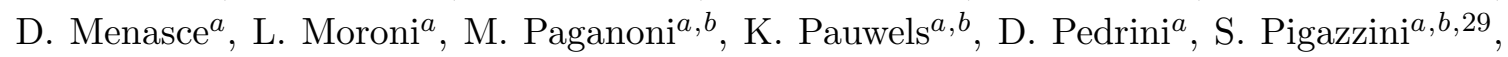

S. Ragazzi ${ }^{a, b}$, T. Tabarelli de Fatis ${ }^{a, b}$

INFN Sezione di Napoli ${ }^{a}$, Università di Napoli 'Federico II' ${ }^{b}$, Napoli, Italy, Università della Basilicata ${ }^{c}$, Potenza, Italy, Università G. Marconi ${ }^{d}$, Roma, Italy

S. Buontempo ${ }^{a}$, N. Cavallo ${ }^{a, c}$, S. Di Guida ${ }^{a, d, 14}$, F. Fabozzi ${ }^{a, c}$, F. Fienga ${ }^{a, b}$, A.O.M. Iorio ${ }^{a, b}$, W.A. $\operatorname{Khan}^{a}$, L. Lista ${ }^{a}$, S. Meola ${ }^{a, d, 14}$, P. Paolucci ${ }^{a, 14}$, C. Sciacca ${ }^{a, b}$, F. Thyssen ${ }^{a}$

INFN Sezione di Padova ${ }^{a}$, Università di Padova ${ }^{b}$, Padova, Italy, Università di Trento ${ }^{c}$, Trento, Italy

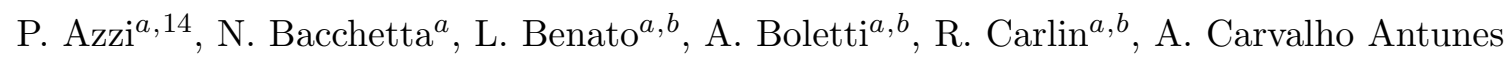
De Oliveira ${ }^{a, b}$, P. Checchia ${ }^{a}$, M. Dall'Osso ${ }^{a, b}$, P. De Castro Manzano $^{a}$, T. Dorigo ${ }^{a}$, U. Dosselli ${ }^{a}$, F. Gasparini ${ }^{a, b}$, U. Gasparini ${ }^{a, b}$, A. Gozzelino ${ }^{a}$, S. Lacaprara ${ }^{a}$, P. Lujan, M. Margoni ${ }^{a, b}$, A.T. Meneguzzo ${ }^{a, b}$, N. Pozzobon ${ }^{a, b}$, P. Ronchese ${ }^{a, b}$, R. Rossin ${ }^{a, b}$,

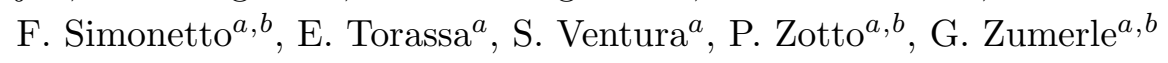

INFN Sezione di Pavia ${ }^{a}$, Università di Pavia ${ }^{b}$, Pavia, Italy

A. Braghieri ${ }^{a}$, A. Magnani ${ }^{a, b}$, P. Montagna ${ }^{a, b}$, S.P. Ratti ${ }^{a, b}$, V. Re ${ }^{a}$, M. Ressegotti,

C. Riccardi ${ }^{a}, b$, P. Salvini ${ }^{a}$, I. Vai ${ }^{a, b}$, P. Vitulo ${ }^{a, b}$

INFN Sezione di Perugia ${ }^{a}$, Università di Perugia ${ }^{b}$, Perugia, Italy

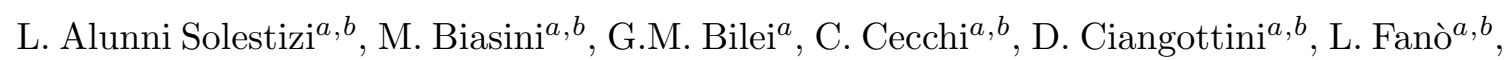
P. Lariccia ${ }^{a, b}$, R. Leonardi ${ }^{a, b}$, E. Manoni $^{a}$, G. Mantovani ${ }^{a, b}$, V. Mariani $^{a, b}$, M. Menichelli $^{a}$, A. $\operatorname{Rossi}^{a, b}$, A. Santocchia ${ }^{a, b}$, D. Spiga ${ }^{a}$

INFN Sezione di Pisa ${ }^{a}$, Università di Pisa ${ }^{b}$, Scuola Normale Superiore di Pisa ${ }^{c}$, Pisa, Italy

K. Androsov ${ }^{a}$, P. Azzurri ${ }^{a, 14}$, G. Bagliesi ${ }^{a}$, J. Bernardini ${ }^{a}$, T. Boccali ${ }^{a}$, L. Borrello,

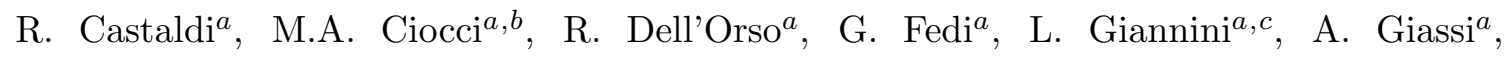
M.T. Grippo ${ }^{a, 28}$, F. Ligabue ${ }^{a, c}$, T. Lomtadze ${ }^{a}$, E. Manca ${ }^{a, c}$, G. Mandorli ${ }^{a, c}$, L. Martini $^{a, b}$, A. Messineo ${ }^{a, b}$, F. Palla ${ }^{a}$, A. Rizzi ${ }^{a, b}$, A. Savoy-Navarro ${ }^{a, 30}$, P. Spagnolo $^{a}$, R. Tenchini ${ }^{a}$, G. Tonelli ${ }^{a, b}$, A. Venturi ${ }^{a}$, P.G. Verdini ${ }^{a}$ 
INFN Sezione di Roma ${ }^{a}$, Sapienza Università di Roma ${ }^{b}$, Rome, Italy

L. Barone ${ }^{a, b}$, F. Cavallari ${ }^{a}$, M. Cipriani ${ }^{a, b}$, D. Del Re ${ }^{a, b, 14}$, E. Di Marco ${ }^{a, b}$, M. Diemoz ${ }^{a}$, S. Gelli ${ }^{a, b}$, E. Longo ${ }^{a, b}$, F. Margaroli ${ }^{a, b}$, B. Marzocchi ${ }^{a, b}$, P. Meridiani $^{a}$, G. Organtini ${ }^{a, b}$,

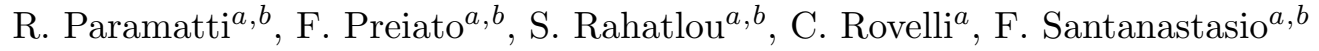

INFN Sezione di Torino ${ }^{a}$, Università di Torino ${ }^{b}$, Torino, Italy, Università del Piemonte Orientale ${ }^{c}$, Novara, Italy

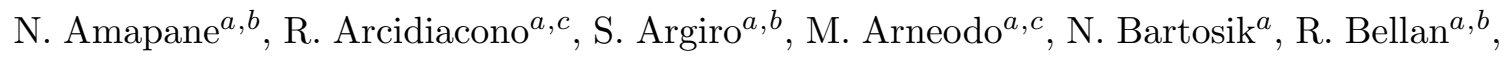

C. Biino ${ }^{a}$, N. Cartiglia ${ }^{a}$, F. Cenna ${ }^{a, b}$, M. Costa ${ }^{a, b}$, R. Covarelli ${ }^{a, b}$, A. Degano ${ }^{a, b}$,

N. Demaria ${ }^{a}$, B. Kiani ${ }^{a, b}$, C. Mariotti ${ }^{a}$, S. Maselli ${ }^{a}$, E. Migliore ${ }^{a, b}$, V. Monaco ${ }^{a}, b$,

E. Monteil ${ }^{a, b}$, M. Monteno $^{a}$, M.M. Obertino ${ }^{a, b}$, L. Pacher $^{a, b}$, N. Pastrone $^{a}$, M. Pelliccioni ${ }^{a}$, G.L. Pinna Angioni ${ }^{a, b}$, F. Ravera ${ }^{a, b}$, A. Romero ${ }^{a, b}$, M. Ruspa ${ }^{a, c}$, R. Sacchi ${ }^{a, b}$,

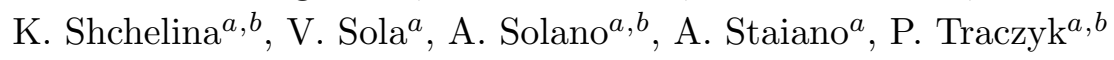

INFN Sezione di Trieste ${ }^{a}$, Università di Trieste ${ }^{b}$, Trieste, Italy

S. Belforte ${ }^{a}$, M. Casarsa ${ }^{a}$, F. Cossutti ${ }^{a}$, G. Della Ricca ${ }^{a, b}$, A. Zanetti ${ }^{a}$

Kyungpook National University, Daegu, Korea

D.H. Kim, G.N. Kim, M.S. Kim, J. Lee, S. Lee, S.W. Lee, C.S. Moon, Y.D. Oh, S. Sekmen, D.C. Son, Y.C. Yang

Chonbuk National University, Jeonju, Korea

A. Lee

Chonnam National University, Institute for Universe and Elementary Particles, Kwangju, Korea

H. Kim, D.H. Moon, G. Oh

Hanyang University, Seoul, Korea

J.A. Brochero Cifuentes, J. Goh, T.J. Kim

Korea University, Seoul, Korea

S. Cho, S. Choi, Y. Go, D. Gyun, S. Ha, B. Hong, Y. Jo, Y. Kim, K. Lee, K.S. Lee, S. Lee, J. Lim, S.K. Park, Y. Roh

Seoul National University, Seoul, Korea

J. Almond, J. Kim, J.S. Kim, H. Lee, K. Lee, K. Nam, S.B. Oh, B.C. Radburn-Smith, S.h. Seo, U.K. Yang, H.D. Yoo, G.B. Yu

University of Seoul, Seoul, Korea

M. Choi, H. Kim, J.H. Kim, J.S.H. Lee, I.C. Park

Sungkyunkwan University, Suwon, Korea

Y. Choi, C. Hwang, J. Lee, I. Yu

Vilnius University, Vilnius, Lithuania

V. Dudenas, A. Juodagalvis, J. Vaitkus 
National Centre for Particle Physics, Universiti Malaya, Kuala Lumpur, Malaysia

I. Ahmed, Z.A. Ibrahim, M.A.B. Md Ali ${ }^{31}$, F. Mohamad Idris ${ }^{32}$, W.A.T. Wan Abdullah, M.N. Yusli, Z. Zolkapli

Centro de Investigacion y de Estudios Avanzados del IPN, Mexico City, Mexico Reyes-Almanza, R, Ramirez-Sanchez, G., Duran-Osuna, M. C., H. Castilla-Valdez, E. De La Cruz-Burelo, I. Heredia-De La Cruz ${ }^{33}$, Rabadan-Trejo, R. I., R. Lopez-Fernandez, J. Mejia Guisao, A. Sanchez-Hernandez

Universidad Iberoamericana, Mexico City, Mexico

S. Carrillo Moreno, C. Oropeza Barrera, F. Vazquez Valencia

Benemerita Universidad Autonoma de Puebla, Puebla, Mexico

I. Pedraza, H.A. Salazar Ibarguen, C. Uribe Estrada

Universidad Autónoma de San Luis Potosí, San Luis Potosí, Mexico

A. Morelos Pineda

University of Auckland, Auckland, New Zealand

D. Krofcheck

University of Canterbury, Christchurch, New Zealand

P.H. Butler

National Centre for Physics, Quaid-I-Azam University, Islamabad, Pakistan

A. Ahmad, M. Ahmad, Q. Hassan, H.R. Hoorani, A. Saddique, M.A. Shah, M. Shoaib, M. Waqas

National Centre for Nuclear Research, Swierk, Poland

H. Bialkowska, M. Bluj, B. Boimska, T. Frueboes, M. Górski, M. Kazana, K. Nawrocki, M. Szleper, P. Zalewski

Institute of Experimental Physics, Faculty of Physics, University of Warsaw, Warsaw, Poland

K. Bunkowski, A. Byszuk ${ }^{34}$, K. Doroba, A. Kalinowski, M. Konecki, J. Krolikowski, M. Misiura, M. Olszewski, A. Pyskir, M. Walczak

Laboratório de Instrumentação e Física Experimental de Partículas, Lisboa, Portugal

P. Bargassa, C. Beirão Da Cruz E Silva, A. Di Francesco, P. Faccioli, M. Gallinaro,

J. Hollar, N. Leonardo, L. Lloret Iglesias, M.V. Nemallapudi, J. Seixas, O. Toldaiev,

D. Vadruccio, J. Varela

Joint Institute for Nuclear Research, Dubna, Russia

S. Afanasiev, P. Bunin, M. Gavrilenko, I. Golutvin, I. Gorbunov, A. Kamenev, V. Karjavin, A. Lanev, A. Malakhov, V. Matveev ${ }^{35,36}$, V. Palichik, V. Perelygin, S. Shmatov, S. Shulha, N. Skatchkov, V. Smirnov, N. Voytishin, A. Zarubin 
Petersburg Nuclear Physics Institute, Gatchina (St. Petersburg), Russia

Y. Ivanov, V. Kim ${ }^{37}$, E. Kuznetsova ${ }^{38}$, P. Levchenko, V. Murzin, V. Oreshkin, I. Smirnov, V. Sulimov, L. Uvarov, S. Vavilov, A. Vorobyev

Institute for Nuclear Research, Moscow, Russia

Yu. Andreev, A. Dermenev, S. Gninenko, N. Golubev, A. Karneyeu, M. Kirsanov, N. Krasnikov, A. Pashenkov, D. Tlisov, A. Toropin

Institute for Theoretical and Experimental Physics, Moscow, Russia

V. Epshteyn, V. Gavrilov, N. Lychkovskaya, V. Popov, I. Pozdnyakov, G. Safronov, A. Spiridonov, A. Stepennov, M. Toms, E. Vlasov, A. Zhokin

Moscow Institute of Physics and Technology, Moscow, Russia

T. Aushev, A. Bylinkin ${ }^{36}$

National Research Nuclear University 'Moscow Engineering Physics Institute' (MEPhI), Moscow, Russia

M. Chadeeva ${ }^{39}$, P. Parygin, D. Philippov, S. Polikarpov, E. Popova, V. Rusinov

P.N. Lebedev Physical Institute, Moscow, Russia

V. Andreev, M. Azarkin ${ }^{36}$, I. Dremin ${ }^{36}$, M. Kirakosyan ${ }^{36}$, A. Terkulov

Skobeltsyn Institute of Nuclear Physics, Lomonosov Moscow State University, Moscow, Russia

A. Baskakov, A. Belyaev, E. Boos, V. Bunichev, M. Dubinin ${ }^{40}$, L. Dudko, A. Ershov, A. Gribushin, V. Klyukhin, O. Kodolova, I. Lokhtin, I. Miagkov, S. Obraztsov, S. Petrushanko, V. Savrin

Novosibirsk State University (NSU), Novosibirsk, Russia

V. Blinov ${ }^{41}$, Y.Skovpen ${ }^{41}$, D. Shtol ${ }^{41}$

State Research Center of Russian Federation, Institute for High Energy Physics, Protvino, Russia

I. Azhgirey, I. Bayshev, S. Bitioukov, D. Elumakhov, V. Kachanov, A. Kalinin, D. Konstantinov, V. Krychkine, V. Petrov, R. Ryutin, A. Sobol, S. Troshin, N. Tyurin, A. Uzunian, A. Volkov

University of Belgrade, Faculty of Physics and Vinca Institute of Nuclear Sciences, Belgrade, Serbia

P. Adzic ${ }^{42}$, P. Cirkovic, D. Devetak, M. Dordevic, J. Milosevic, V. Rekovic

Centro de Investigaciones Energéticas Medioambientales y Tecnológicas (CIEMAT), Madrid, Spain

J. Alcaraz Maestre, M. Barrio Luna, M. Cerrada, N. Colino, B. De La Cruz, A. Delgado Peris, A. Escalante Del Valle, C. Fernandez Bedoya, J.P. Fernández Ramos, J. Flix, M.C. Fouz, P. Garcia-Abia, O. Gonzalez Lopez, S. Goy Lopez, J.M. Hernandez, M.I. Josa, A. Pérez-Calero Yzquierdo, J. Puerta Pelayo, A. Quintario Olmeda, I. Redondo, L. Romero, M.S. Soares, A. Álvarez Fernández 
Universidad Autónoma de Madrid, Madrid, Spain

J.F. de Trocóniz, M. Missiroli, D. Moran

Universidad de Oviedo, Oviedo, Spain

J. Cuevas, C. Erice, J. Fernandez Menendez, I. Gonzalez Caballero, J.R. González Fernández, E. Palencia Cortezon, S. Sanchez Cruz, I. Suárez Andrés, P. Vischia, J.M. Vizan Garcia

Instituto de Física de Cantabria (IFCA), CSIC-Universidad de Cantabria, Santander, Spain

I.J. Cabrillo, A. Calderon, B. Chazin Quero, E. Curras, J. Duarte Campderros, M. Fernandez, J. Garcia-Ferrero, G. Gomez, A. Lopez Virto, J. Marco, C. Martinez Rivero, P. Martinez Ruiz del Arbol, F. Matorras, J. Piedra Gomez, T. Rodrigo, A. Ruiz-Jimeno, L. Scodellaro, N. Trevisani, I. Vila, R. Vilar Cortabitarte

\section{CERN, European Organization for Nuclear Research, Geneva, Switzerland}

D. Abbaneo, E. Auffray, P. Baillon, A.H. Ball, D. Barney, M. Bianco, P. Bloch, A. Bocci, C. Botta, T. Camporesi, R. Castello, M. Cepeda, G. Cerminara, E. Chapon, Y. Chen, D. d'Enterria, A. Dabrowski, V. Daponte, A. David, M. De Gruttola, A. De Roeck, M. Dobson, B. Dorney, T. du Pree, M. Dünser, N. Dupont, A. Elliott-Peisert, P. Everaerts, F. Fallavollita, G. Franzoni, J. Fulcher, W. Funk, D. Gigi, K. Gill, F. Glege, D. Gulhan, P. Harris, J. Hegeman, V. Innocente, P. Janot, O. Karacheban ${ }^{17}$, J. Kieseler, H. Kirschenmann, V. Knünz, A. Kornmayer ${ }^{14}$, M.J. Kortelainen, M. Krammer ${ }^{1}$, C. Lange, P. Lecoq, C. Lourenço, M.T. Lucchini, L. Malgeri, M. Mannelli, A. Martelli, F. Meijers, J.A. Merlin, S. Mersi, E. Meschi, P. Milenovic ${ }^{43}$, F. Moortgat, M. Mulders, H. Neugebauer, S. Orfanelli, L. Orsini, L. Pape, E. Perez, M. Peruzzi, A. Petrilli, G. Petrucciani, A. Pfeiffer, M. Pierini, A. Racz, T. Reis, G. Rolandi4 ${ }^{44}$ M. Rovere, H. Sakulin, C. Schäfer, C. Schwick, M. Seidel, M. Selvaggi, A. Sharma, P. Silva, P. Sphicas ${ }^{45}$, A. Stakia, J. Steggemann, M. Stoye, M. Tosi, D. Treille, A. Triossi, A. Tsirou, V. Veckalns ${ }^{46}$, M. Verweij, W.D. Zeuner

\section{Paul Scherrer Institut, Villigen, Switzerland}

W. Bertl ${ }^{\dagger}$, L. Caminada ${ }^{47}$, K. Deiters, W. Erdmann, R. Horisberger, Q. Ingram, H.C. Kaestli, D. Kotlinski, U. Langenegger, T. Rohe, S.A. Wiederkehr

\section{Institute for Particle Physics, ETH Zurich, Zurich, Switzerland}

F. Bachmair, L. Bäni, P. Berger, L. Bianchini, B. Casal, G. Dissertori, M. Dittmar, M. Donegà, C. Grab, C. Heidegger, D. Hits, J. Hoss, G. Kasieczka, T. Klijnsma, W. Lustermann, B. Mangano, M. Marionneau, M.T. Meinhard, D. Meister, F. Micheli, P. Musella, F. Nessi-Tedaldi, F. Pandolfi, J. Pata, F. Pauss, G. Perrin, L. Perrozzi, M. Quittnat, M. Reichmann, M. Schönenberger, L. Shchutska, V.R. Tavolaro, K. Theofilatos, M.L. Vesterbacka Olsson, R. Wallny, D.H. Zhu

\section{Universität Zürich, Zurich, Switzerland}

T.K. Aarrestad, C. Amsler ${ }^{48}$, M.F. Canelli, A. De Cosa, R. Del Burgo, S. Donato, C. Galloni, T. Hreus, B. Kilminster, J. Ngadiuba, D. Pinna, G. Rauco, P. Robmann, D. Salerno, C. Seitz, Y. Takahashi, A. Zucchetta 
National Central University, Chung-Li, Taiwan

V. Candelise, T.H. Doan, Sh. Jain, R. Khurana, C.M. Kuo, W. Lin, A. Pozdnyakov, S.S. Yu

National Taiwan University (NTU), Taipei, Taiwan

Arun Kumar, P. Chang, Y. Chao, K.F. Chen, P.H. Chen, F. Fiori, W.-S. Hou, Y. Hsiung, Y.F. Liu, R.-S. Lu, E. Paganis, A. Psallidas, A. Steen, J.f. Tsai

Chulalongkorn University, Faculty of Science, Department of Physics, Bangkok, Thailand

B. Asavapibhop, K. Kovitanggoon, G. Singh, N. Srimanobhas

Çukurova University, Physics Department, Science and Art Faculty, Adana, Turkey

A. Adiguzel ${ }^{49}$, F. Boran, S. Cerci ${ }^{50}$, S. Damarseckin, Z.S. Demiroglu, C. Dozen, I. Dumanoglu, S. Girgis, G. Gokbulut, Y. Guler, I. Hos ${ }^{51}$, E.E. Kangal ${ }^{52}$, O. Kara, A. Kayis Topaksu, U. Kiminsu, M. Oglakci, G. Onengut ${ }^{53}$, K. Ozdemir ${ }^{54}$, D. Sunar $\mathrm{Cerci}^{50}$, B. Tali ${ }^{50}$, S. Turkcapar, I.S. Zorbakir, C. Zorbilmez

Middle East Technical University, Physics Department, Ankara, Turkey

B. Bilin, G. Karapinar ${ }^{55}$, K. Ocalan ${ }^{56}$, M. Yalvac, M. Zeyrek

Bogazici University, Istanbul, Turkey

E. Gülmez, M. Kaya ${ }^{57}$, O. Kaya ${ }^{58}$, S. Tekten, E.A. Yetkin ${ }^{59}$

Istanbul Technical University, Istanbul, Turkey

M.N. Agaras, S. Atay, A. Cakir, K. Cankocak

Institute for Scintillation Materials of National Academy of Science of Ukraine, Kharkov, Ukraine

B. Grynyov

National Scientific Center, Kharkov Institute of Physics and Technology, Kharkov, Ukraine

L. Levchuk, P. Sorokin

University of Bristol, Bristol, United Kingdom

R. Aggleton, F. Ball, L. Beck, J.J. Brooke, D. Burns, E. Clement, D. Cussans, O. Davignon,

H. Flacher, J. Goldstein, M. Grimes, G.P. Heath, H.F. Heath, J. Jacob, L. Kreczko,

C. Lucas, D.M. Newbold ${ }^{60}$, S. Paramesvaran, A. Poll, T. Sakuma, S. Seif El Nasr-storey,

D. Smith, V.J. Smith

Rutherford Appleton Laboratory, Didcot, United Kingdom

K.W. Bell, A. Belyaev ${ }^{61}$, C. Brew, R.M. Brown, L. Calligaris, D. Cieri, D.J.A. Cockerill, J.A. Coughlan, K. Harder, S. Harper, E. Olaiya, D. Petyt, C.H. Shepherd-Themistocleous,

A. Thea, I.R. Tomalin, T. Williams

Imperial College, London, United Kingdom

G. Auzinger, R. Bainbridge, S. Breeze, O. Buchmuller, A. Bundock, S. Casasso, M. Citron,

D. Colling, L. Corpe, P. Dauncey, G. Davies, A. De Wit, M. Della Negra, R. Di Maria, 
A. Elwood, Y. Haddad, G. Hall, G. Iles, T. James, R. Lane, C. Laner, L. Lyons, A.-M. Magnan, S. Malik, L. Mastrolorenzo, T. Matsushita, J. Nash, A. Nikitenko ${ }^{6}$, V. Palladino, M. Pesaresi, D.M. Raymond, A. Richards, A. Rose, E. Scott, C. Seez, A. Shtipliyski, S. Summers, A. Tapper, K. Uchida, M. Vazquez Acosta ${ }^{62}$, T. Virdee ${ }^{14}$, N. Wardle, D. Winterbottom, J. Wright, S.C. Zenz

Brunel University, Uxbridge, United Kingdom

J.E. Cole, P.R. Hobson, A. Khan, P. Kyberd, I.D. Reid, P. Symonds, L. Teodorescu, M. Turner

Baylor University, Waco, U.S.A.

A. Borzou, K. Call, J. Dittmann, K. Hatakeyama, H. Liu, N. Pastika, C. Smith

Catholic University of America, Washington DC, U.S.A.

R. Bartek, A. Dominguez

The University of Alabama, Tuscaloosa, U.S.A.

A. Buccilli, S.I. Cooper, C. Henderson, P. Rumerio, C. West

Boston University, Boston, U.S.A.

D. Arcaro, A. Avetisyan, T. Bose, D. Gastler, D. Rankin, C. Richardson, J. Rohlf, L. Sulak, D. Zou

Brown University, Providence, U.S.A.

G. Benelli, D. Cutts, A. Garabedian, J. Hakala, U. Heintz, J.M. Hogan, K.H.M. Kwok, E. Laird, G. Landsberg, Z. Mao, M. Narain, J. Pazzini, S. Piperov, S. Sagir, R. Syarif, D. $\mathrm{Yu}$

University of California, Davis, Davis, U.S.A.

R. Band, C. Brainerd, D. Burns, M. Calderon De La Barca Sanchez, M. Chertok, J. Conway, R. Conway, P.T. Cox, R. Erbacher, C. Flores, G. Funk, M. Gardner, W. Ko, R. Lander, C. Mclean, M. Mulhearn, D. Pellett, J. Pilot, S. Shalhout, M. Shi, J. Smith, M. Squires, D. Stolp, K. Tos, M. Tripathi, Z. Wang

University of California, Los Angeles, U.S.A.

M. Bachtis, C. Bravo, R. Cousins, A. Dasgupta, A. Florent, J. Hauser, M. Ignatenko, N. Mccoll, D. Saltzberg, C. Schnaible, V. Valuev

University of California, Riverside, Riverside, U.S.A.

E. Bouvier, K. Burt, R. Clare, J. Ellison, J.W. Gary, S.M.A. Ghiasi Shirazi, G. Hanson, J. Heilman, P. Jandir, E. Kennedy, F. Lacroix, O.R. Long, M. Olmedo Negrete, M.I. Paneva, A. Shrinivas, W. Si, L. Wang, H. Wei, S. Wimpenny, B. R. Yates

\section{University of California, San Diego, La Jolla, U.S.A.}

J.G. Branson, S. Cittolin, M. Derdzinski, B. Hashemi, A. Holzner, D. Klein, G. Kole, V. Krutelyov, J. Letts, I. Macneill, M. Masciovecchio, D. Olivito, S. Padhi, M. Pieri, M. Sani, V. Sharma, S. Simon, M. Tadel, A. Vartak, S. Wasserbaech ${ }^{63}$, J. Wood, F. Würthwein, A. Yagil, G. Zevi Della Porta 
University of California, Santa Barbara - Department of Physics, Santa Barbara, U.S.A.

N. Amin, R. Bhandari, J. Bradmiller-Feld, C. Campagnari, A. Dishaw, V. Dutta, M. Franco Sevilla, C. George, F. Golf, L. Gouskos, J. Gran, R. Heller, J. Incandela, S.D. Mullin, A. Ovcharova, H. Qu, J. Richman, D. Stuart, I. Suarez, J. Yoo

California Institute of Technology, Pasadena, U.S.A.

D. Anderson, J. Bendavid, A. Bornheim, J.M. Lawhorn, H.B. Newman, T. Nguyen, C. Pena, M. Spiropulu, J.R. Vlimant, S. Xie, Z. Zhang, R.Y. Zhu

Carnegie Mellon University, Pittsburgh, U.S.A.

M.B. Andrews, T. Ferguson, T. Mudholkar, M. Paulini, J. Russ, M. Sun, H. Vogel, I. Vorobiev, M. Weinberg

University of Colorado Boulder, Boulder, U.S.A.

J.P. Cumalat, W.T. Ford, F. Jensen, A. Johnson, M. Krohn, S. Leontsinis, T. Mulholland, K. Stenson, S.R. Wagner

Cornell University, Ithaca, U.S.A.

J. Alexander, J. Chaves, J. Chu, S. Dittmer, K. Mcdermott, N. Mirman, J.R. Patterson, A. Rinkevicius, A. Ryd, L. Skinnari, L. Soffi, S.M. Tan, Z. Tao, J. Thom, J. Tucker, P. Wittich, M. Zientek

\section{Fermi National Accelerator Laboratory, Batavia, U.S.A.}

S. Abdullin, M. Albrow, G. Apollinari, A. Apresyan, A. Apyan, S. Banerjee, L.A.T. Bauerdick, A. Beretvas, J. Berryhill, P.C. Bhat, G. Bolla ${ }^{\dagger}$, K. Burkett, J.N. Butler, A. Canepa, G.B. Cerati, H.W.K. Cheung, F. Chlebana, M. Cremonesi, J. Duarte, V.D. Elvira, J. Freeman, Z. Gecse, E. Gottschalk, L. Gray, D. Green, S. Grünendahl, O. Gutsche, R.M. Harris, S. Hasegawa, J. Hirschauer, Z. Hu, B. Jayatilaka, S. Jindariani, M. Johnson, U. Joshi, B. Klima, B. Kreis, S. Lammel, D. Lincoln, R. Lipton, M. Liu, T. Liu, R. Lopes De Sá, J. Lykken, K. Maeshima, N. Magini, J.M. Marraffino, S. Maruyama, D. Mason, P. McBride, P. Merkel, S. Mrenna, S. Nahn, V. O’Dell, K. Pedro, O. Prokofyev, G. Rakness, L. Ristori, B. Schneider, E. Sexton-Kennedy, A. Soha, W.J. Spalding, L. Spiegel, S. Stoynev, J. Strait, N. Strobbe, L. Taylor, S. Tkaczyk, N.V. Tran, L. Uplegger, E.W. Vaandering, C. Vernieri, M. Verzocchi, R. Vidal, M. Wang, H.A. Weber, A. Whitbeck University of Florida, Gainesville, U.S.A.

D. Acosta, P. Avery, P. Bortignon, D. Bourilkov, A. Brinkerhoff, A. Carnes, M. Carver, D. Curry, R.D. Field, I.K. Furic, J. Konigsberg, A. Korytov, K. Kotov, P. Ma, K. Matchev, H. Mei, G. Mitselmakher, D. Rank, D. Sperka, N. Terentyev, L. Thomas, J. Wang, S. Wang, J. Yelton

Florida International University, Miami, U.S.A.

Y.R. Joshi, S. Linn, P. Markowitz, J.L. Rodriguez

Florida State University, Tallahassee, U.S.A.

A. Ackert, T. Adams, A. Askew, S. Hagopian, V. Hagopian, K.F. Johnson, T. Kolberg, G. Martinez, T. Perry, H. Prosper, A. Saha, A. Santra, R. Yohay 
Florida Institute of Technology, Melbourne, U.S.A.

M.M. Baarmand, V. Bhopatkar, S. Colafranceschi, M. Hohlmann, D. Noonan, T. Roy, F. Yumiceva

University of Illinois at Chicago (UIC), Chicago, U.S.A.

M.R. Adams, L. Apanasevich, D. Berry, R.R. Betts, R. Cavanaugh, X. Chen, O. Evdokimov, C.E. Gerber, D.A. Hangal, D.J. Hofman, K. Jung, J. Kamin, I.D. Sandoval Gonzalez, M.B. Tonjes, H. Trauger, N. Varelas, H. Wang, Z. Wu, J. Zhang

The University of Iowa, Iowa City, U.S.A.

B. Bilki ${ }^{64}$, W. Clarida, K. Dilsiz ${ }^{65}$, S. Durgut, R.P. Gandrajula, M. Haytmyradov, V. Khristenko, J.-P. Merlo, H. Mermerkaya ${ }^{66}$, A. Mestvirishvili, A. Moeller, J. Nachtman, H. Ogul ${ }^{67}$, Y. Onel, F. Ozok ${ }^{68}$, A. Penzo, C. Snyder, E. Tiras, J. Wetzel, K. Yi

Johns Hopkins University, Baltimore, U.S.A.

B. Blumenfeld, A. Cocoros, N. Eminizer, D. Fehling, L. Feng, A.V. Gritsan, P. Maksimovic, J. Roskes, U. Sarica, M. Swartz, M. Xiao, C. You

The University of Kansas, Lawrence, U.S.A.

A. Al-bataineh, P. Baringer, A. Bean, S. Boren, J. Bowen, J. Castle, S. Khalil, A. Kropivnitskaya, D. Majumder, W. Mcbrayer, M. Murray, C. Royon, S. Sanders, E. Schmitz, R. Stringer, J.D. Tapia Takaki, Q. Wang

Kansas State University, Manhattan, U.S.A.

A. Ivanov, K. Kaadze, Y. Maravin, A. Mohammadi, L.K. Saini, N. Skhirtladze, S. Toda

Lawrence Livermore National Laboratory, Livermore, U.S.A.

F. Rebassoo, D. Wright

University of Maryland, College Park, U.S.A.

C. Anelli, A. Baden, O. Baron, A. Belloni, B. Calvert, S.C. Eno, C. Ferraioli, N.J. Hadley, S. Jabeen, G.Y. Jeng, R.G. Kellogg, J. Kunkle, A.C. Mignerey, F. Ricci-Tam, Y.H. Shin, A. Skuja, S.C. Tonwar

\section{Massachusetts Institute of Technology, Cambridge, U.S.A.}

D. Abercrombie, B. Allen, V. Azzolini, R. Barbieri, A. Baty, R. Bi, S. Brandt, W. Busza, I.A. Cali, M. D'Alfonso, Z. Demiragli, G. Gomez Ceballos, M. Goncharov, D. Hsu, Y. Iiyama, G.M. Innocenti, M. Klute, D. Kovalskyi, Y.S. Lai, Y.-J. Lee, A. Levin, P.D. Luckey, B. Maier, A.C. Marini, C. Mcginn, C. Mironov, S. Narayanan, X. Niu, C. Paus, C. Roland, G. Roland, J. Salfeld-Nebgen, G.S.F. Stephans, K. Tatar, D. Velicanu, J. Wang, T.W. Wang, B. Wyslouch

University of Minnesota, Minneapolis, U.S.A.

A.C. Benvenuti, R.M. Chatterjee, A. Evans, P. Hansen, S. Kalafut, Y. Kubota, Z. Lesko, J. Mans, S. Nourbakhsh, N. Ruckstuhl, R. Rusack, J. Turkewitz

University of Mississippi, Oxford, U.S.A.

J.G. Acosta, S. Oliveros 
University of Nebraska-Lincoln, Lincoln, U.S.A.

E. Avdeeva, K. Bloom, D.R. Claes, C. Fangmeier, R. Gonzalez Suarez, R. Kamalieddin, I. Kravchenko, J. Monroy, J.E. Siado, G.R. Snow, B. Stieger

State University of New York at Buffalo, Buffalo, U.S.A.

M. Alyari, J. Dolen, A. Godshalk, C. Harrington, I. Iashvili, D. Nguyen, A. Parker, S. Rappoccio, B. Roozbahani

Northeastern University, Boston, U.S.A.

G. Alverson, E. Barberis, A. Hortiangtham, A. Massironi, D.M. Morse, D. Nash, T. Orimoto, R. Teixeira De Lima, D. Trocino, D. Wood

Northwestern University, Evanston, U.S.A.

S. Bhattacharya, O. Charaf, K.A. Hahn, N. Mucia, N. Odell, B. Pollack, M.H. Schmitt, K. Sung, M. Trovato, M. Velasco

University of Notre Dame, Notre Dame, U.S.A.

N. Dev, M. Hildreth, K. Hurtado Anampa, C. Jessop, D.J. Karmgard, N. Kellams, K. Lannon, N. Loukas, N. Marinelli, F. Meng, C. Mueller, Y. Musienko ${ }^{35}$, M. Planer, A. Reinsvold, R. Ruchti, G. Smith, S. Taroni, M. Wayne, M. Wolf, A. Woodard

The Ohio State University, Columbus, U.S.A.

J. Alimena, L. Antonelli, B. Bylsma, L.S. Durkin, S. Flowers, B. Francis, A. Hart, C. Hill, W. Ji, B. Liu, W. Luo, D. Puigh, B.L. Winer, H.W. Wulsin

Princeton University, Princeton, U.S.A.

A. Benaglia, S. Cooperstein, O. Driga, P. Elmer, J. Hardenbrook, P. Hebda, S. Higginbotham, D. Lange, J. Luo, D. Marlow, K. Mei, I. Ojalvo, J. Olsen, C. Palmer, P. Piroué, D. Stickland, C. Tully

University of Puerto Rico, Mayaguez, U.S.A.

S. Malik, S. Norberg

Purdue University, West Lafayette, U.S.A.

A. Barker, V.E. Barnes, S. Das, S. Folgueras, L. Gutay, M.K. Jha, M. Jones, A.W. Jung, A. Khatiwada, D.H. Miller, N. Neumeister, C.C. Peng, J.F. Schulte, J. Sun, F. Wang, W. Xie

Purdue University Northwest, Hammond, U.S.A.

T. Cheng, N. Parashar, J. Stupak

Rice University, Houston, U.S.A.

A. Adair, B. Akgun, Z. Chen, K.M. Ecklund, F.J.M. Geurts, M. Guilbaud, W. Li, B. Michlin, M. Northup, B.P. Padley, J. Roberts, J. Rorie, Z. Tu, J. Zabel

University of Rochester, Rochester, U.S.A.

A. Bodek, P. de Barbaro, R. Demina, Y.t. Duh, T. Ferbel, M. Galanti, A. Garcia-Bellido, J. Han, O. Hindrichs, A. Khukhunaishvili, K.H. Lo, P. Tan, M. Verzetti 
The Rockefeller University, New York, U.S.A.

R. Ciesielski, K. Goulianos, C. Mesropian

Rutgers, The State University of New Jersey, Piscataway, U.S.A.

A. Agapitos, J.P. Chou, Y. Gershtein, T.A. Gómez Espinosa, E. Halkiadakis, M. Heindl,

E. Hughes, S. Kaplan, R. Kunnawalkam Elayavalli, S. Kyriacou, A. Lath, R. Montalvo,

K. Nash, M. Osherson, H. Saka, S. Salur, S. Schnetzer, D. Sheffield, S. Somalwar, R. Stone,

S. Thomas, P. Thomassen, M. Walker

University of Tennessee, Knoxville, U.S.A.

A.G. Delannoy, M. Foerster, J. Heideman, G. Riley, K. Rose, S. Spanier, K. Thapa

Texas A\&M University, College Station, U.S.A.

O. Bouhali ${ }^{69}$, A. Castaneda Hernandez ${ }^{69}$, A. Celik, M. Dalchenko, M. De Mattia, A. Delgado, S. Dildick, R. Eusebi, J. Gilmore, T. Huang, T. Kamon ${ }^{70}$, R. Mueller, Y. Pakhotin, R. Patel, A. Perloff, L. Perniè, D. Rathjens, A. Safonov, A. Tatarinov, K.A. Ulmer

\section{Texas Tech University, Lubbock, U.S.A.}

N. Akchurin, J. Damgov, F. De Guio, P.R. Dudero, J. Faulkner, E. Gurpinar, S. Kunori, K. Lamichhane, S.W. Lee, T. Libeiro, T. Peltola, S. Undleeb, I. Volobouev, Z. Wang

Vanderbilt University, Nashville, U.S.A.

S. Greene, A. Gurrola, R. Janjam, W. Johns, C. Maguire, A. Melo, H. Ni, P. Sheldon, S. Tuo, J. Velkovska, Q. Xu

University of Virginia, Charlottesville, U.S.A.

M.W. Arenton, P. Barria, B. Cox, R. Hirosky, A. Ledovskoy, H. Li, C. Neu, T. Sinthuprasith, X. Sun, Y. Wang, E. Wolfe, F. Xia

Wayne State University, Detroit, U.S.A.

R. Harr, P.E. Karchin, J. Sturdy, S. Zaleski

University of Wisconsin - Madison, Madison, WI, U.S.A.

M. Brodski, J. Buchanan, C. Caillol, S. Dasu, L. Dodd, S. Duric, B. Gomber, M. Grothe, M. Herndon, A. Hervé, U. Hussain, P. Klabbers, A. Lanaro, A. Levine, K. Long, R. Loveless, G.A. Pierro, G. Polese, T. Ruggles, A. Savin, N. Smith, W.H. Smith, D. Taylor, N. Woods

$\dagger$ : Deceased

1: Also at Vienna University of Technology, Vienna, Austria

2: Also at State Key Laboratory of Nuclear Physics and Technology, Peking University, Beijing, China

3: Also at Universidade Estadual de Campinas, Campinas, Brazil

4: Also at Universidade Federal de Pelotas, Pelotas, Brazil

5: Also at Université Libre de Bruxelles, Bruxelles, Belgium

6: Also at Institute for Theoretical and Experimental Physics, Moscow, Russia

7: Also at Joint Institute for Nuclear Research, Dubna, Russia

8: Also at Suez University, Suez, Egypt 
9: Now at British University in Egypt, Cairo, Egypt

10: Now at Helwan University, Cairo, Egypt

11: Also at Université de Haute Alsace, Mulhouse, France

12: Also at Skobeltsyn Institute of Nuclear Physics, Lomonosov Moscow State University, Moscow, Russia

13: Also at Tbilisi State University, Tbilisi, Georgia

14: Also at CERN, European Organization for Nuclear Research, Geneva, Switzerland

15: Also at RWTH Aachen University, III. Physikalisches Institut A, Aachen, Germany

16: Also at University of Hamburg, Hamburg, Germany

17: Also at Brandenburg University of Technology, Cottbus, Germany

18: Also at MTA-ELTE Lendület CMS Particle and Nuclear Physics Group, Eötvös Loránd University, Budapest, Hungary

19: Also at Institute of Nuclear Research ATOMKI, Debrecen, Hungary

20: Also at Institute of Physics, University of Debrecen, Debrecen, Hungary

21: Also at Indian Institute of Technology Bhubaneswar, Bhubaneswar, India

22: Also at Institute of Physics, Bhubaneswar, India

23: Also at University of Visva-Bharati, Santiniketan, India

24: Also at University of Ruhuna, Matara, Sri Lanka

25: Also at Isfahan University of Technology, Isfahan, Iran

26: Also at Yazd University, Yazd, Iran

27: Also at Plasma Physics Research Center, Science and Research Branch, Islamic Azad University, Tehran, Iran

28: Also at Università degli Studi di Siena, Siena, Italy

29: Also at INFN Sezione di Milano-Bicocca; Università di Milano-Bicocca, Milano, Italy

30: Also at Purdue University, West Lafayette, U.S.A.

31: Also at International Islamic University of Malaysia, Kuala Lumpur, Malaysia

32: Also at Malaysian Nuclear Agency, MOSTI, Kajang, Malaysia

33: Also at Consejo Nacional de Ciencia y Tecnología, Mexico city, Mexico

34: Also at Warsaw University of Technology, Institute of Electronic Systems, Warsaw, Poland

35: Also at Institute for Nuclear Research, Moscow, Russia

36: Now at National Research Nuclear University 'Moscow Engineering Physics Institute' (MEPhI), Moscow, Russia

37: Also at St. Petersburg State Polytechnical University, St. Petersburg, Russia

38: Also at University of Florida, Gainesville, U.S.A.

39: Also at P.N. Lebedev Physical Institute, Moscow, Russia

40: Also at California Institute of Technology, Pasadena, U.S.A.

41: Also at Budker Institute of Nuclear Physics, Novosibirsk, Russia

42: Also at Faculty of Physics, University of Belgrade, Belgrade, Serbia

43: Also at University of Belgrade, Faculty of Physics and Vinca Institute of Nuclear Sciences, Belgrade, Serbia

44: Also at Scuola Normale e Sezione dell'INFN, Pisa, Italy

45: Also at National and Kapodistrian University of Athens, Athens, Greece

46: Also at Riga Technical University, Riga, Latvia

47: Also at Universität Zürich, Zurich, Switzerland

48: Also at Stefan Meyer Institute for Subatomic Physics (SMI), Vienna, Austria

49: Also at Istanbul University, Faculty of Science, Istanbul, Turkey

50: Also at Adiyaman University, Adiyaman, Turkey

51: Also at Istanbul Aydin University, Istanbul, Turkey 
52: Also at Mersin University, Mersin, Turkey

53: Also at Cag University, Mersin, Turkey

54: Also at Piri Reis University, Istanbul, Turkey

55: Also at Izmir Institute of Technology, Izmir, Turkey

56: Also at Necmettin Erbakan University, Konya, Turkey

57: Also at Marmara University, Istanbul, Turkey

58: Also at Kafkas University, Kars, Turkey

59: Also at Istanbul Bilgi University, Istanbul, Turkey

60: Also at Rutherford Appleton Laboratory, Didcot, United Kingdom

61: Also at School of Physics and Astronomy, University of Southampton, Southampton, United Kingdom

62: Also at Instituto de Astrofísica de Canarias, La Laguna, Spain

63: Also at Utah Valley University, Orem, U.S.A.

64: Also at Beykent University, Istanbul, Turkey

65: Also at Bingol University, Bingol, Turkey

66: Also at Erzincan University, Erzincan, Turkey

67: Also at Sinop University, Sinop, Turkey

68: Also at Mimar Sinan University, Istanbul, Istanbul, Turkey

69: Also at Texas A\&M University at Qatar, Doha, Qatar

70: Also at Kyungpook National University, Daegu, Korea 